\title{
H $\alpha$ DOTS: A CATALOG OF FAINT EMISSION-LINE OBJECTS DISCOVERED IN NARROWBAND IMAGES
}

\author{
Jessica A. Kellar ${ }^{1}$, John J. Salzer ${ }^{2}$, Gary Wegner ${ }^{1}$, Caryl Gronwall ${ }^{3}$, and Anna Williams ${ }^{2,4}$ \\ ${ }^{1}$ Department of Physics \& Astronomy, 6127 Wilder Laboratory, Dartmouth College, Hanover, NH 03755, USA; \\ jessica.a.kellar@dartmouth.edu,gaw@northstar.dartmouth.edu \\ 2 Department of Astronomy, Indiana University, 727 East Third Street, Bloomington, IN 47405, USA; slaz@ astro.indiana.edu \\ ${ }^{3}$ Department of Astronomy \& Astrophysics, Pennsylvania State University, 525 Davey Lab, University Park, PA 16802, USA; caryl@astro.psu.edu

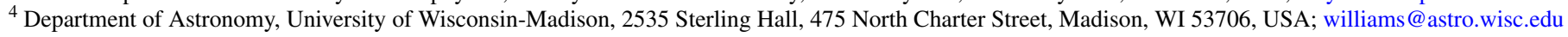 \\ Received 2011 August 2; accepted 2012 April 13; published 2012 May 14
}

\begin{abstract}
During a wide-field narrowband $\mathrm{H} \alpha$ imaging survey, we noted the presence of numerous isolated emission-line point sources in the data. These objects could represent ultra-low-luminosity galaxies at low-redshift (detection via $\mathrm{H} \alpha$ ), isolated extragalactic $\mathrm{H}$ II regions associated with the galaxy targeted by the original observation, or background galaxies or QSOs where strong emission lines (most often [O III] $\lambda 5007$ ) redshift into our narrowband filter. We have carried out a systematic search for these "H $\alpha$ dots" in over $20015 \times 15$ arcmin fields. To date we have cataloged 61 candidate emission-line sources in roughly $11.7 \mathrm{deg}^{2}$. The sample has a median $R$ magnitude of 19.5 , and detects objects as faint as $R=22.6$. Follow-up spectroscopy reveals that $\sim 85 \%$ of the candidates are bona fide emission-line objects, with roughly $60 \%$ of the real sources being lower-redshift objects (detection via H $\alpha$ ) and $40 \%$ being higher-redshift objects detected via [O III] emission or some other emission line. Here we present the results of our initial survey and follow-up spectroscopy. We use our sample to study the properties (including star-formation rates and metal abundances) of low-luminosity star-forming galaxies in the nearby universe and of low-metallicity star-forming galaxies at $z \approx 0.33$.
\end{abstract}

Key words: galaxies: abundances - galaxies: dwarf - galaxies: starburst - surveys

Online-only material: color figure

\section{INTRODUCTION}

Studying star formation is fundamental to our understanding of galaxy formation and evolution. Large-scale star formation typically takes place in the extended disks of spiral and irregular galaxies (Kennicutt 1998). However, star formation can also take place in low-luminosity dwarf galaxies (Kennicutt et al. 2008; Lee et al. 2007, 2009) and in outlying H II regions (Ferguson et al. 1998; Ryan-Weber et al. 2004; Werk et al. 2008; Boquien et al. 2007; Werk et al. 2010). Also, extended-UV (XUV) emission has been detected in the outskirts of many galaxies (Thilker et al. 2007). Low-luminosity star-forming dwarfs can be used to study starbursts, metallicities of dwarfs, and the effect of environment on star formation in dwarfs. XUV and outlying $\mathrm{H}$ II regions may be associated with ongoing galaxy interactions (e.g., Thilker et al. 2007; Werk et al. 2010). Studying the nature of star formation in the outer regions of galaxies can improve our understanding of the galaxy formation process, the build-up of galaxy disks, and the influence of galaxy interactions on star formation in galaxies. Narrowband searches for isolated H II regions of the type carried out by Ryan-Weber et al. (2004) and Werk et al. (2010) can also lead to the detection of higher-redshift star-forming galaxies and active galactic nuclei (AGNs) selected via other emission lines. These higher-redshift emission-line galaxies (ELGs) tend to have extremely low metallicities (e.g., Kakazu et al. 2007) and provide important insights into ongoing galaxy formation occurring at relatively low redshifts.

We have initiated a survey that attempts to expand on the previous work of Ryan-Weber et al. (2004) and Werk et al. (2010), who performed a systematic search for outlying H II regions in the SINGG (Survey for Ionization in Neutral Gas Galaxies; Meurer et al. 2006) survey, and analyzed their optical and UV properties. Rather than focusing primarily on the detection of isolated extragalactic H II regions, however, our survey attempts to uncover a more generic population of compact emission-line objects identified using a large sample of narrowband $\mathrm{H} \alpha$ images being acquired for an entirely different purpose. Our survey uses narrowband images taken as part of the ALFALFA (Arecibo Legacy Fast ALFA) $\mathrm{H} \alpha$ survey (Sugden et al. 2008; J. J. Salzer et al. 2012, in preparation). This project has the goal of studying the star-formation properties of a volume-limited sample of galaxies and calculating the starformation rate (SFR) density in the local universe.

While analyzing the $\mathrm{H} \alpha$ images obtained for the ALFALFA $\mathrm{H} \alpha$ project, we unexpectedly discovered a number of faint pointlike emission-line sources (hereafter referred to as $\mathrm{H} \alpha$ dots $^{5}$ ) that were located well away from the galaxies originally targeted by the observations. These objects were found via visual inspection of the ALFALFA $\mathrm{H} \alpha$ data following the completion of the image processing. They are very compact sources (essentially point sources) of line emission that remained after the continuum-subtraction process for our narrowband $\mathrm{H} \alpha$ images was complete. Figure 1 shows $R$-band and continuumsubtracted $\mathrm{H} \alpha$ images of the spiral galaxy UGC 2134 (the large galaxy in the center of the frame). The expectation was that by subtracting the properly scaled and PSF-matched $R$-band image from the $\mathrm{H} \alpha$ image, only the $\mathrm{H} \alpha$ emission in the target galaxy would remain. However, two isolated points of emission remain that are clearly distinct from any emission coming from the target galaxy. Subsequent visual searches of additional processed ALFALFA $\mathrm{H} \alpha$ images revealed several more $\mathrm{H} \alpha$ dots.

In order to determine how common these compact emissionline sources are, we decided to carry out a systematic search of

\footnotetext{
5 We use the term $\mathrm{H} \alpha$ dot to describe the emission-line objects found in our survey. We do not intend for this term to be used to refer to the entire class of objects that are compact and possess strong line emission.
} 


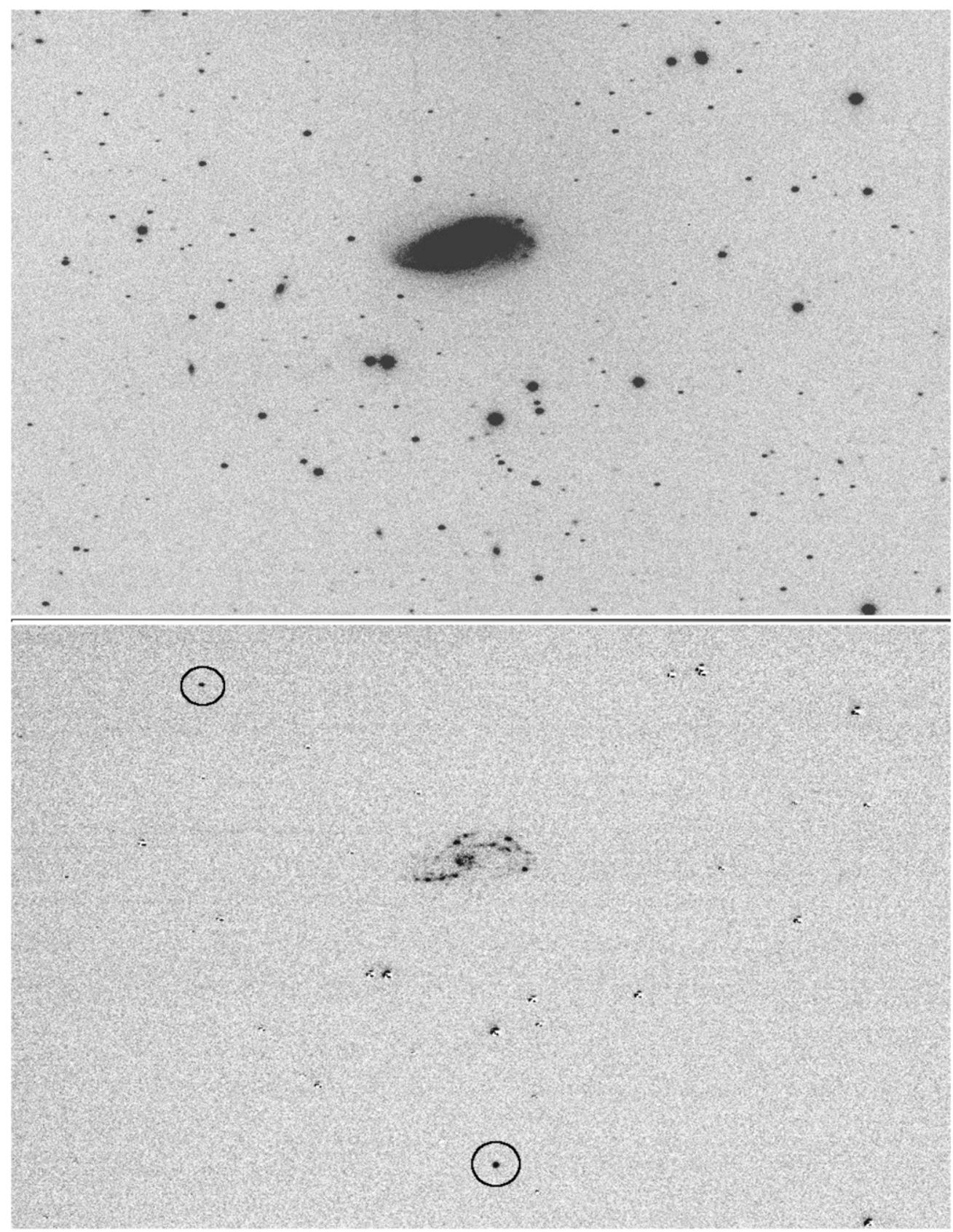

Figure 1. Example of an image showing two newly discovered $\mathrm{H} \alpha$ dots. The top panel is the continuum $R$-band image of UGC 2134 (the spiral in the center of the image), and the lower panel shows the H $\alpha$ continuum-subtracted image of the same field. The two $\mathrm{H} \alpha$ dots in the image are circled. The dots appear as compact and isolated emission-line sources that are well separated from the galaxy that was the target of the $\mathrm{H} \alpha$ observations.

all of the available ALFALFA $\mathrm{H} \alpha$ data. To facilitate our search, as well as to ensure that the selection of candidate objects was done in a uniform way, we developed software to search for $\mathrm{H} \alpha$ dots. Currently we have searched over 200 fields to create our initial catalog of $\mathrm{H} \alpha$ dot candidates. The ALFALFA $\mathrm{H} \alpha$ program is an ongoing survey, and we expect to find more $\mathrm{H} \alpha$ dots as additional observations are obtained.

Detecting point-like sources of emission in narrowband images is just the first step in the discovery of these new and interesting objects. The objects detected via this survey method fall into a number of categories representing quite different astrophysical sources. For example, the emission line detected may be $\mathrm{H} \alpha$, which would place the $\mathrm{H} \alpha$ dot at roughly the same distance as the target ALFALFA $\mathrm{H} \alpha$ galaxy. Given the nature of our selection process (see Section 3), such sources could be either dwarf galaxies with modest to high SFRs or isolated extragalactic $\mathrm{H}$ II regions similar to objects found by Ryan-Weber et al. (2004) and Werk et al. (2010). However, it is common to detect higher-redshift objects via this survey method as well (e.g., Boroson et al. 1993; Kakazu et al. 2007). We might expect to detect galaxies via their [O III] or [O II] emission, as well as high-redshift QSOs via lines like Mg II, [C III], or Ly $\alpha$. In order to distinguish between these various possibilities, one must obtain follow-up spectra to identify the nature of the objects being detected, as well as to determine their physical characteristics. 
In this paper, we present the results of a survey of ALFALFA $\mathrm{H} \alpha$ fields covering $\sim 11.7 \mathrm{deg}^{2}$ for $\mathrm{H} \alpha$ dot candidates. In Section 2 we describe the data used for the $\mathrm{H} \alpha$ dot survey, as well as the preliminary image processing and $\mathrm{H} \alpha$ reductions. In Section 3 we explain the method for finding $\mathrm{H} \alpha$ dots and give an overview of the software developed for the project. In Section 4 we present our first list of $\mathrm{H} \alpha$ dots and describe their observed properties. In Section 5 we discuss the spectroscopic follow-up of the $\mathrm{H} \alpha$ dot candidates and use the spectra to distinguish between higher-redshift star-forming galaxies, AGNs, and lower-redshift star-forming regions. In Section 6 we present a preliminary analysis of the properties of the $\mathrm{H} \alpha$ dots, including luminosities, SFRs, abundances, and clustering properties. We discuss the insights these objects give us about extragalactic H II regions, low-luminosity and low-metallicity dwarf galaxies, and high-redshift metal-poor star-forming galaxies. In Section 7 we summarize the main results of the paper. We use the concordance cosmology of $H_{0}=70 \mathrm{~km} \mathrm{~s}^{-1} \mathrm{Mpc}^{-1}, \Omega_{m}=0.3$, and $\Omega_{\Lambda}=0.7$ (Dunkley et al. 2009).

\section{THE ALFALFA H $\alpha$ SURVEY}

The data used for the $\mathrm{H} \alpha$ dot survey were acquired as part of the ALFALFA $\mathrm{H} \alpha$ survey. ALFALFA $\mathrm{H} \alpha$ is a long-term narrowband imaging survey with the goal of obtaining $\mathrm{H} \alpha$ images for a large ( $\sim 1400$ objects $)$, volume-limited catalog of galaxies in the local universe. The ALFALFA $\mathrm{H} \alpha$ galaxy sample has been selected from the catalog of $\mathrm{H}$ I-detected sources found by the ALFALFA survey, a blind $21 \mathrm{~cm}$ radio survey being carried out with the $305 \mathrm{~m}$ Arecibo radio telescope (Giovanelli et al. 2005). The ALFALFA survey will cover about $7000 \mathrm{deg}^{2}$ of the sky in a redshift range between -1600 and $18,000 \mathrm{~km} \mathrm{~s}^{-1}$ with a velocity resolution of about $5 \mathrm{~km} \mathrm{~s}^{-1}$. Source catalogs for about $40 \%$ of its total sky area have already been published (Haynes et al. 2011), as have a number of key science results (e.g., Martin et al. 2010, 2012).

The ALFALFA H $\alpha$ survey (Sugden et al. 2008; J. J. Salzer et al. 2012, in preparation) is a follow-up optical survey designed to measure the SFR density in the local universe as well as to explore the star-formation properties and modes of a large, representative sample of galaxies. The galaxy target list for the ALFALFA $\mathrm{H} \alpha$ project is selected to fall within the velocity range $1500-7500 \mathrm{~km} \mathrm{~s}^{-1}$. The excellent sensitivity of the ALFALFA survey (e.g., Giovanelli et al. 2005; Haynes et al. 2011), combined with the volume-limited nature of the narrowband galaxy sample, ensures that the ALFALFA $\mathrm{H} \alpha$ data set will be comprehensive and highly complete. As shown in Haynes et al. (2011), the ALFALFA survey method detects objects with $\mathrm{H}$ I masses as low as $6 \times 10^{8} M_{\odot}$ at the outer velocity limit of the ALFALFA $\mathrm{H} \alpha$ study. The deep H i selection method means that the ALFALFA H $\alpha$ sample will include many low surface brightness and dwarf galaxies that are often under-represented in magnitude-limited optical catalogs. This study will complement the nearby 11HUGS sample (Kennicutt et al. 2008; Lee et al. 2007, 2009), which focuses on galaxies within $11 \mathrm{Mpc}$ of the Milky Way.

The galaxies observed for the ALFALFA $\mathrm{H} \alpha$ study were selected from two regions of the ALFALFA survey area. In the north Galactic cap (Spring sky) we selected galaxies located in the declination range 10-16 deg over the full right ascension range of the ALFALFA survey $\left(7^{\mathrm{h}} 30^{\mathrm{m}}-17^{\mathrm{h}} 0^{\mathrm{m}}\right)$, while in the south Galactic cap (Fall sky) the sources were located between declinations 24-29 deg and within the right ascension range $22^{\mathrm{h}}-3^{\mathrm{h}}$. In the Fall region, all ALFALFA detections in the
Table 1

Filter Properties

\begin{tabular}{lccc}
\hline \hline Filter & $\begin{array}{c}\text { Velocity Range } \\
\left(\mathrm{km} \mathrm{s}^{-1}\right)\end{array}$ & $\begin{array}{c}\text { Width } \\
(\AA)\end{array}$ & $\begin{array}{c}\text { Central Wavelength } \\
(\AA)\end{array}$ \\
$(1)$ & $(2)$ & $(3)$ & $(4)$ \\
\hline$R$ & $\ldots$ & 1541 & 6425 \\
HA1 & $0-1460$ & 67 & 6573 \\
HA2 & $1460-3150$ & 74 & 6618 \\
HA3 & $3150-5300$ & 68 & 6659 \\
HA4 & $5300-7810$ & 71 & 6707 \\
\hline
\end{tabular}

velocity range listed above are being observed $(N=543)$ within this contiguous region (area $\sim 340 \mathrm{deg}^{2}$ ). In the Spring area the ALFALFA $\mathrm{H} \alpha$ sample was created by randomly selecting $2 \times 2 \mathrm{deg}$ survey grids out of the $\sim 830 \mathrm{deg}^{2}$ area until the total number of galaxies contained within those grids represented a suitably large but observationally manageable list of targets. The final sample size arrived at via this process was $N=853$. Even though the Spring ALFALFA $\mathrm{H} \alpha$ sample does not cover a contiguous area on the sky, it is still nonetheless a volume-limited sample since every ALFALFA galaxy located in the selected grids will be observed. The ALFALFA H $\alpha$ project was split into two phases based on the velocities of the galaxies. Phase 1 included all galaxies in the designated areas with velocities between 1500 and $5300 \mathrm{~km} \mathrm{~s}^{-1}$, while Phase 2 involved observations of galaxies in the velocity range $5300-7500 \mathrm{~km} \mathrm{~s}^{-1}$. Most of the ALFALFA $\mathrm{H} \alpha$ data used for the current study are from Phase 1 .

The ALFALFA $\mathrm{H} \alpha$ observations consist of images taken through both a continuum $R$-band filter and a set of narrowband $\mathrm{H} \alpha$ filters with the WIYN 0.9 m telescope ${ }^{6}$ at Kitt Peak National Observatory. The proper $\mathrm{H} \alpha$ filter is chosen based on the redshift of the target galaxy, which is known from the ALFALFA H I observations. The velocity range, full width at half-intensity, and central wavelength of the filters used for this project are shown in Table 1. Observations consist of a pair of $1200 \mathrm{~s}$ exposures through the appropriate narrowband filter sandwiched around a single $240 \mathrm{~s}$ image taken through the $R$ filter. The latter is used for continuum subtraction of the narrowband images. The field of view employed with the S2KB CCD detector used on the WIYN $0.9 \mathrm{~m}$ telescope is $15 \times 15$ arcmin. Typically the target galaxy is centered in the middle of the image and each image covers a much larger portion of the sky than just the target galaxy. Most of the current phase of the ALFALFA $\mathrm{H} \alpha$ project makes use of the HA2 and HA3 filters; a small number of fields have been imaged using HA1 and HA4.

The image processing and measurement is done using the Image Reduction and Analysis Facility ${ }^{7}$ (IRAF). Standard reduction methods are employed. We remove the mean bias level using the overscan region of each image, subtract a mean bias image to remove any two-dimensional bias structure, then divide each image by a scaled dome flat-field image to correct for pixel-to-pixel sensitivity variations. The images are cleaned of cosmic rays using the LACOS_IM software (van Dokkum 2001). Next the three images for a given target are aligned

\footnotetext{
6 The $0.9 \mathrm{~m}$ telescope is operated by WIYN Inc. on behalf of a Consortium of nine partner Universities and Organizations (see www.noao.edu/0.9m/ partners). WIYN is a joint partnership of the University of Wisconsin at Madison, Indiana University, Yale University, and the National Optical Astronomical Observatory.

7 IRAF is distributed by the National Optical Astronomy Observatory, which is operated by the Association of Universities for Research in Astronomy (AURA) under cooperative agreement with the National Science Foundation.
} 
to a common coordinate system, and an astrometric solution is applied. Finally, the $R$-band image is scaled to match the intensity levels of the $\mathrm{H} \alpha$ images, after which it is subtracted from each $\mathrm{H} \alpha$ image and the two resulting continuum-subtracted $\mathrm{H} \alpha$ images are combined. At this point the processed $\mathrm{H} \alpha$ images are ready for measurement. Fluxes derived from the ALFALFA $\mathrm{H} \alpha$ images are calibrated using observations of spectrophotometric standard stars (Oke \& Gunn 1983) to a precision of $1 \%-2 \%$ or better.

\section{SELECTING H $\alpha$ DOT CANDIDATES}

As described in the Introduction, the first few $\mathrm{H} \alpha$ dots were found by visual examination of some of the processed narrowband images taken for the ALFALFA $\mathrm{H} \alpha$ project. As we developed a growing interest in mining our extensive data set of $\mathrm{H} \alpha$ images for additional $\mathrm{H} \alpha$ dots, we decided to undertake a rigorous and systematic search. In order to carry out a search that was reasonably complete and statistically meaningful, we needed to develop software to automate the selection process. We also needed to clearly define the characteristics of the objects we would be cataloging.

For the purpose of our study, $\mathrm{H} \alpha$ dots have three primary distinguishing characteristics. First, they must possess significant line emission. Despite the name we have chosen to refer to them by, there is no requirement that the emission line present be $\mathrm{H} \alpha$. As we shall see in subsequent sections of this paper, the majority of the $\mathrm{H} \alpha$ dots are detected via $\mathrm{H} \alpha$ emission, but many of the dots exhibit strong emission from some other line (e.g., [O III] $\lambda 5007, \mathrm{Mg}_{\text {II }} \lambda 2798$, etc.). Second, the dots must appear as compact sources. We do not require that the dots be point sources, but select against objects that are obviously extended. This selection criterion is clearly somewhat subjective. However, we justify it by noting the large range of point-spread function (PSF) FWHMs present in our narrowband survey data. With stellar PSFs in our data ranging from 1.0 arcsec to greater than 3 arcsec, a given object may or may not be resolved depending on which end of the PSF distribution it was observed. Hence, we adopted the subjective criterion of compactness rather than the more precise criterion of unresolved. Since our goal is to discover and catalog interesting and unique objects, we feel that this "softer" selection characteristic better serves our needs. Finally, we require that each $\mathrm{H} \alpha$ dot is isolated, in the sense that it is not contained within a larger galaxy. In practice, this means that the $\mathrm{H} \alpha$ dots are located outside an isophotal radius corresponding roughly to an $R$-band surface brightness level of $24 \mathrm{mag} \operatorname{arcsec}^{-2}$. The vast majority of detected $\mathrm{H} \alpha$ dots are actually located very far from the target ALFALFA galaxy in the images (e.g., the two objects illustrated in Figure 1 are typical). This criterion means that we do not select H II regions within large spiral or irregular galaxies, which are quite common in our data. Such objects are both compact and possess strong emission, but are not the types of objects we are interested in. While our software is very effective at detecting and cataloging large numbers of $\mathrm{H}$ II regions in the galaxies in our fields, these objects are not the focus of our current study.

To carry out the search for $\mathrm{H} \alpha$ dots, we developed a software package to identify a complete and objectively selected list of objects from our narrowband $\mathrm{H} \alpha$ images. Our detection software is written in the IRAF scripting language, and makes use of existing IRAF routines, as well as scripts previously developed by the authors, to streamline the selection process. The scripts take as input the fully reduced and processed ALFALFA $\mathrm{H} \alpha$ images described in the previous section. The software is designed to be sensitive to very weak sources, and detects emission-line sources regardless of their morphology. The methodology is fairly straightforward. First the software uses a slightly modified version of DAOFIND to locate all objects present in the combined image for each field. For this step, we sum the aligned $R$ and $\mathrm{H} \alpha$ images to give us the deepest possible image. In this case the $\mathrm{H} \alpha$ images used are those with the continuum light still present. The detected sources are cataloged and accurate astrometry is determined for each object. Next, each object is photometered in the $R$ and $\mathrm{H} \alpha$ images using a small circular aperture. The instrumental magnitudes are then used to construct a diagnostic diagram that is used to select $\mathrm{H} \alpha$ dot candidates.

$\mathrm{H} \alpha$ dots are selected based on two criteria: the difference between the instrumental $R$ and $\mathrm{H} \alpha$ magnitudes $(\Delta \mathrm{m})$, and the ratio of this magnitude difference to the uncertainty in the magnitude difference. These criteria are similar to, but not identical with, the ones used by Ryan-Weber et al. (2004) and Werk et al. (2010) in their studies. Using the small-aperture photometry measured for each object in the image, calculating the magnitude difference is straightforward:

$$
\Delta m=m_{\mathrm{H} \alpha}-m_{R} .
$$

Since the $R$-band image is photometrically scaled to the final $\mathrm{H} \alpha$ image during the ALFALFA $\mathrm{H} \alpha$ processing, objects with $\Delta \mathrm{m}=0$ have no emission present in the narrowband filter. Objects with negative values of $\Delta m$ are potential $\mathrm{H} \alpha$ dot candidates. The magnitude difference error is calculated from the $\mathrm{H} \alpha$ and $R$ magnitude errors using

$$
\sigma_{\Delta_{m}}=\left(\sigma_{\mathrm{H} \alpha}^{2}+\sigma_{R}^{2}\right)^{1 / 2}
$$

A good way to measure the significance of a possible detection is the ratio of the magnitude difference to the magnitude difference error:

$$
\text { ratio }=\Delta \mathrm{m} / \sigma_{\Delta_{\mathrm{m}}}
$$

This is effectively a signal-to-noise measurement for a possible emission-line detection.

Values for $\Delta m, \sigma_{\Delta_{m}}$, and ratio are calculated for every object in the field. These are used to generate two diagnostic plots of the type shown in Figure 2. One plot shows $\Delta m$ versus instrumental $R$ magnitude and the other plot is $\Delta m$ versus ratio. Objects with larger ratio and more negative $\Delta m$ are more likely to be $\mathrm{H} \alpha$ dots. The user is allowed to set limits for $\Delta m$ and ratio, based on the appearance of the diagnostic plot. The selected objects are then inspected visually to ensure that they appear to be real sources. The user is presented with a mosaic of image cutouts centered on the putative dot ( $R$-band image, $\mathrm{H} \alpha$ images-both continuum-subtracted and unsubtracted) that allows them to evaluate the reality of each object. Examples of three such mosaics are shown in Figure 3. At this stage the user accepts or rejects each candidate, after which they label them either as $\mathrm{H} \alpha$ dots (if suitably isolated) or H II regions in a galaxy. The H II regions are cataloged in a separate process, and are available for further study if so desired. The current paper focuses solely on the $\mathrm{H} \alpha$ dots.

Establishing reasonable limits for $\Delta m$ and ratio for inclusion or exclusion of objects from our $\mathrm{H} \alpha$ dot catalog was the focus of much work. Our goal was to set the detection limits to select candidate objects as faint as possible, while minimizing the number of false detections. We also wanted to use a single set of limits for constructing our catalog of $\mathrm{H} \alpha$ dots, rather than 


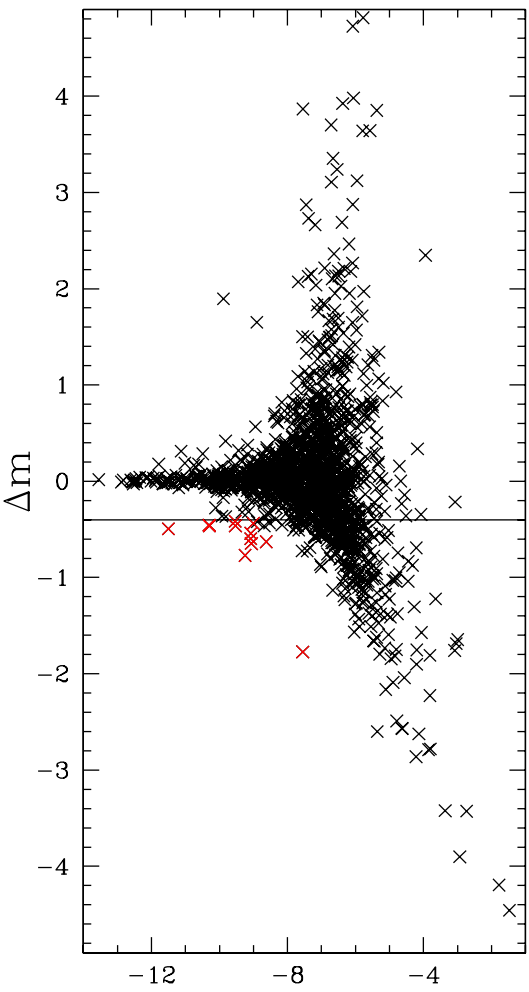

Instrumental $\mathrm{R}$ Magnitude

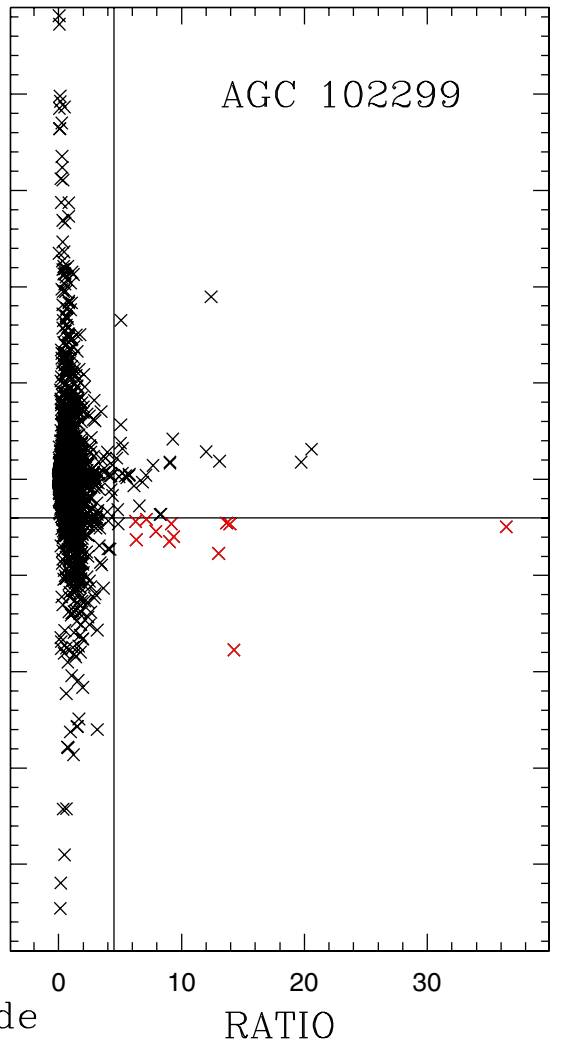

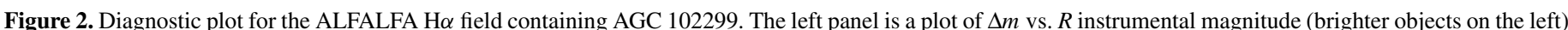

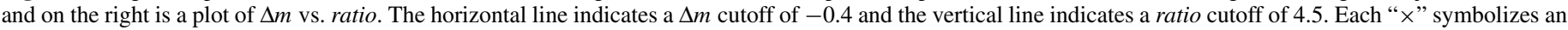

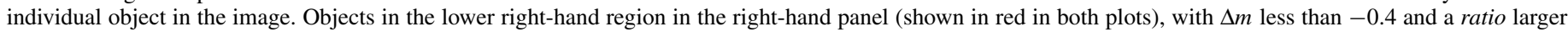
than 4.5 , are potential $\mathrm{H} \alpha$ dot candidates.

(A color version of this figure is available in the online journal.)

varying the limits from field to field. Therefore, we experimented with a range of values for $\Delta m$ and ratio, and ran tests on numerous fields before reaching a decision on what the limits should be. In addition, we used our experience with previous emission-line surveys (e.g., Boroson et al. 1993; Salzer et al. $2000,2001)$ as a guide for reasonable values for the limits. We also used the results of our first set of follow-up spectra of $\sim 30$ $\mathrm{H} \alpha$ dots from our initial searches (see below) to inform our final decisions and selection methods. At the end of this exercise, we chose to use $\Delta m=-0.4$ and ratio $=4.5$. All subsequent searches of the ALFALFA $\mathrm{H} \alpha$ data for $\mathrm{H} \alpha$ dots have used these values.

We note that the value of $\Delta m=-0.4$ corresponds to $f_{\mathrm{H} \alpha} / f_{\text {cont }}$ $\approx 0.45$, which implies an equivalent width selection limit of $\sim 30 \AA$ given the typical narrowband filter width of $\sim 70 \AA$. Although both the $\Delta m$ and ratio limits are specific to the current survey project, we suggest that these values could be translated (in an approximate sense) to limits of other, similar surveys. For example, the equivalent width limit mentioned above is likely to be typical of what one would achieve with similar emissionline detection studies. Furthermore, the limiting value of the ratio term of 4.5 is close to the $\mathrm{S} / \mathrm{N}$ selection limit of 5.0 used by the KISS objective-prism emission-line survey (Salzer et al. 2000, 2001). Even though the survey methods are completely different, the data yield similar detection limits.

After the final selection of the $\mathrm{H} \alpha$ dots is completed, the fluxes of all sources are measured in both the narrowband and broadband images using synthetic aperture photometry (we employ the IRAF PHOT task for this function). The apertures used are optimized for each source, and are typically small (10 arcsec diameters or less). Accurate photometric calibrations are available as part of the ALFALFA $\mathrm{H} \alpha$ project.

\section{FIRST LIST OF H $\alpha$ DOT CANDIDATES}

We ran our search software on 205 narrowband $\mathrm{H} \alpha$ images in order to develop an initial list of $\mathrm{H} \alpha$ dot candidates. This represents all of the ALFALFA $\mathrm{H} \alpha$ project data obtained for the first five observing runs, representing data obtained between 2006 March and 2007 October. The total area covered by this first $\mathrm{H} \alpha$ dot list is $11.694 \mathrm{deg}^{2}$. We found $61 \mathrm{H} \alpha$ dot candidates in total, resulting in a surface density of 5.22 objects deg $^{-2}$. However, there are only 60 unique objects included in this number. One object, cataloged as both $\mathrm{H} \alpha$ dot 7 and $\mathrm{H} \alpha$ dot 24 , represents independent detections of the same object found in two fields observed on different observing runs. The two fields happen to overlap in the area where the emission-line source is located. We decided to retain both detections of this source for completeness. However, it appears only once in all subsequent figures and analyses of the $\mathrm{H} \alpha$ dot sample.

Table 2 presents our first list of $\mathrm{H} \alpha$ dot candidates. The sources are grouped by the ALFALFA $\mathrm{H} \alpha$ observing run from which the narrowband images originate, and are then ordered by right ascension within these groups. Hence, objects 1-13 are from the 2006 March ALFALFA H $\alpha$ run, objects 14-22 from 2006 September, objects 23-28 from 2007 February, objects 29-42 from 2007 May, and objects 43-61 from 2007 October. The table includes the $\mathrm{H} \alpha$ dot number (assigned via the scheme described above), right ascension and declination 
Table 2

$\mathrm{H} \alpha$ Dot Candidates-Observed Properties

\begin{tabular}{|c|c|c|c|c|c|c|}
\hline $\begin{array}{l}\text { H } \alpha \text { Dot } \\
\text { (1) }\end{array}$ & $\begin{array}{l}\text { R.A. } \\
(\mathrm{J} 2000) \\
(2)\end{array}$ & $\begin{array}{c}\text { Decl. } \\
(\mathrm{J} 2000) \\
(3)\end{array}$ & $\begin{array}{l}\Delta m \\
(4)\end{array}$ & $\begin{array}{l}\text { Ratio } \\
\text { (5) }\end{array}$ & $\begin{array}{c}\text { Emission-line Flux } \\
\left(\times 10^{-14} \mathrm{erg} \mathrm{s}^{-1} \mathrm{~cm}^{-2}\right) \\
(6)\end{array}$ & $\begin{array}{c}R \\
(\mathrm{mag}) \\
(7)\end{array}$ \\
\hline 1 & $8: 47: 08.5$ & 10:10:36.2 & -0.61 & 5.47 & $0.112 \pm 0.020$ & $20.12 \pm 0.12$ \\
\hline 2 & $8: 47: 15.3$ & 10:02:30.4 & -1.11 & 30.52 & $1.163 \pm 0.032$ & $18.61 \pm 0.05$ \\
\hline 3 & $8: 47: 17.6$ & 10:02:36.7 & -1.01 & 19.87 & $0.967 \pm 0.046$ & $19.23 \pm 0.05$ \\
\hline 4 & $8: 47: 17.9$ & 10:02:33.5 & -1.63 & 90.69 & $5.147 \pm 0.042$ & $17.12 \pm 0.02$ \\
\hline 5 & $10: 36: 59.6$ & 12:06:07.6 & -0.55 & 7.61 & $0.173 \pm 0.022$ & $19.90 \pm 0.09$ \\
\hline 6 & $10: 37: 09.5$ & $12: 14: 56.9$ & -0.78 & 4.73 & $0.075 \pm 0.023$ & $20.72 \pm 0.23$ \\
\hline 7 & 10:37:11.2 & 12:07:48.4 & -2.37 & 5.43 & $0.102 \pm 0.022$ & $22.64 \pm 0.47$ \\
\hline 8 & $11: 40: 48.8$ & 13:07:40.5 & -0.72 & 9.47 & $0.463 \pm 0.074$ & $15.90 \pm 0.02$ \\
\hline 9 & $12: 12: 37.8$ & 11:03:29.1 & -0.42 & 4.81 & $0.066 \pm 0.019$ & $19.98 \pm 0.09$ \\
\hline 10 & $12: 16: 55.3$ & 12:50:06.5 & -0.59 & 13.77 & $0.434 \pm 0.045$ & $18.33 \pm 0.05$ \\
\hline 11 & $12: 39: 00.4$ & $13: 23: 55.4$ & -1.59 & 6.80 & $0.222 \pm 0.031$ & $21.18 \pm 0.25$ \\
\hline 12 & $12: 50: 55.3$ & 12:01:31.0 & -0.73 & 18.79 & $0.820 \pm 0.043$ & $18.06 \pm 0.02$ \\
\hline 13 & $12: 59: 59.9$ & $13: 36: 45.0$ & -0.57 & 14.22 & $0.373 \pm 0.032$ & $18.24 \pm 0.04$ \\
\hline 14 & $23: 11: 56.1$ & $27: 22: 12.0$ & -0.61 & 10.54 & $0.350 \pm 0.037$ & $18.84 \pm 0.05$ \\
\hline 15 & $0: 42: 55.6$ & $25: 51: 25.9$ & -0.65 & 5.77 & $0.221 \pm 0.054$ & $19.84 \pm 0.09$ \\
\hline 16 & $0: 45: 14.2$ & $27: 27: 13.9$ & -0.50 & 9.50 & $0.466 \pm 0.038$ & $18.28 \pm 0.03$ \\
\hline 17 & $1: 44: 47.3$ & $27: 16: 39.0$ & -0.63 & 4.76 & $0.158 \pm 0.028$ & $19.84 \pm 0.08$ \\
\hline 18 & $1: 50: 37.7$ & $27: 33: 42.9$ & -0.68 & 10.57 & $0.357 \pm 0.055$ & $18.37 \pm 0.05$ \\
\hline 19 & $2: 34: 34.2$ & $27: 16: 54.4$ & -1.41 & 5.59 & $0.496 \pm 0.083$ & $20.96 \pm 0.19$ \\
\hline 20 & $2: 38: 50.1$ & $27: 47: 43.4$ & -1.21 & 34.17 & $1.199 \pm 0.043$ & $18.25 \pm 0.04$ \\
\hline 21 & $2: 39: 04.2$ & $27: 52: 36.7$ & -1.24 & 19.51 & $0.912 \pm 0.059$ & $19.44 \pm 0.09$ \\
\hline 22 & $2: 39: 12.6$ & $27: 52: 02.3$ & -1.07 & 4.53 & $0.110 \pm 0.035$ & $20.89 \pm 0.19$ \\
\hline 23 & 10:06:51.7 & $12: 37: 30.9$ & -0.50 & 8.60 & $0.078 \pm 0.011$ & $20.13 \pm 0.03$ \\
\hline 24 & $10: 37: 11.3$ & $12: 07: 49.4$ & -2.14 & 4.81 & $0.092 \pm 0.019$ & $22.64 \pm 0.41$ \\
\hline 25 & $10: 48: 38.0$ & 12:17:07.9 & -1.14 & 5.58 & $0.091 \pm 0.031$ & $21.07 \pm 0.23$ \\
\hline 26 & 10:48:55.2 & 12:10:52.1 & -0.94 & 6.17 & $0.163 \pm 0.041$ & $20.68 \pm 0.19$ \\
\hline 27 & $10: 49: 06.8$ & $12: 16: 49.6$ & -0.49 & 6.21 & $0.174 \pm 0.049$ & $19.49 \pm 0.16$ \\
\hline 28 & 12:01:23.7 & 14:00:39.5 & -1.87 & 6.37 & $0.156 \pm 0.018$ & $22.13 \pm 0.38$ \\
\hline 29 & 11:41:19.7 & 16:03:22.0 & -0.44 & 4.98 & $0.090 \pm 0.019$ & $20.00 \pm 0.13$ \\
\hline 30 & $11: 41: 55.6$ & $15: 56: 35.6$ & -0.68 & 8.48 & $0.222 \pm 0.048$ & $19.57 \pm 0.06$ \\
\hline 31 & $13: 57: 05.3$ & 14:04:50.9 & -1.50 & 23.89 & $1.010 \pm 0.040$ & $19.07 \pm 0.08$ \\
\hline 32 & 14:04:57.9 & 12:09:18.7 & -0.41 & 10.12 & $0.295 \pm 0.041$ & $18.17 \pm 0.05$ \\
\hline 33 & $14: 05: 24.6$ & 12:10:15.8 & -0.48 & 6.98 & $0.244 \pm 0.058$ & $17.58 \pm 0.04$ \\
\hline 34 & $14: 30: 43.9$ & 13:57:07.8 & -0.58 & 25.65 & $1.018 \pm 0.040$ & $17.45 \pm 0.03$ \\
\hline 35 & 16:04:17.0 & $11: 37: 32.5$ & -0.53 & 18.02 & $0.560 \pm 0.029$ & $17.99 \pm 0.05$ \\
\hline 36 & $16: 05: 21.8$ & $13: 42: 04.9$ & -0.49 & 15.46 & $0.388 \pm 0.033$ & $18.56 \pm 0.04$ \\
\hline 37 & 16:08:37.1 & 11:59:01.4 & -1.37 & 20.29 & $0.286 \pm 0.020$ & $20.09 \pm 0.06$ \\
\hline 38 & $16: 26: 22.7$ & 11:29:03.6 & -0.42 & 8.19 & $0.265 \pm 0.042$ & $18.91 \pm 0.04$ \\
\hline 39 & $16: 26: 43.8$ & $11: 37: 51.7$ & -0.75 & 7.68 & $0.202 \pm 0.028$ & $19.47 \pm 0.08$ \\
\hline 40 & $16: 26: 44.2$ & $11: 37: 46.4$ & -1.33 & 10.28 & $0.267 \pm 0.027$ & $20.45 \pm 0.11$ \\
\hline 41 & $16: 26: 53.6$ & 11:39:09.0 & -0.62 & 96.52 & $4.392 \pm 0.076$ & $15.83 \pm 0.02$ \\
\hline 42 & $16: 27: 04.6$ & 11:37:48.0 & -0.86 & 13.78 & $0.435 \pm 0.036$ & $19.26 \pm 0.06$ \\
\hline 43 & $23: 18: 43.1$ & $26: 13: 35.6$ & -0.84 & 21.53 & $0.425 \pm 0.027$ & $18.59 \pm 0.03$ \\
\hline 44 & $23: 21: 17.8$ & $27: 54: 31.9$ & -0.56 & 18.34 & $0.481 \pm 0.031$ & $18.29 \pm 0.04$ \\
\hline 45 & $23: 40: 54.0$ & $27: 27: 20.9$ & -0.60 & 4.52 & $0.095 \pm 0.023$ & $20.31 \pm 0.14$ \\
\hline 46 & 0:38:16.1 & $26: 59: 31.3$ & -0.48 & 8.26 & $0.287 \pm 0.067$ & $18.81 \pm 0.05$ \\
\hline 47 & $0: 40: 38.0$ & 27:02:30.4 & -1.75 & 4.88 & $0.255 \pm 0.043$ & $21.83 \pm 0.24$ \\
\hline 48 & $0: 40: 54.3$ & $26: 57: 21.1$ & -0.48 & 14.09 & $0.574 \pm 0.049$ & $17.98 \pm 0.03$ \\
\hline 49 & $0: 41: 02.7$ & $25: 27: 42.5$ & -0.52 & 19.28 & $0.659 \pm 0.034$ & $18.44 \pm 0.02$ \\
\hline 50 & $0: 41: 40.3$ & $25: 26: 51.6$ & -0.92 & 4.53 & $0.074 \pm 0.019$ & $21.53 \pm 0.16$ \\
\hline 51 & $0: 43: 52.5$ & $26: 08: 56.9$ & -0.50 & 6.52 & $0.289 \pm 0.062$ & $19.84 \pm 0.07$ \\
\hline 52 & $0: 47: 13.2$ & $26: 40: 21.6$ & -0.44 & 4.62 & $0.061 \pm 0.015$ & $20.30 \pm 0.06$ \\
\hline 53 & $0: 49: 44.9$ & $27: 07: 52.5$ & -0.85 & 56.91 & $1.653 \pm 0.032$ & $17.57 \pm 0.02$ \\
\hline 54 & $0: 55: 43.2$ & $24: 16: 51.4$ & -0.59 & 4.71 & $0.074 \pm 0.016$ & $20.64 \pm 0.12$ \\
\hline 55 & $1: 44: 40.3$ & $27: 54: 35.5$ & -2.01 & 4.83 & $0.091 \pm 0.019$ & $22.38 \pm 0.31$ \\
\hline 56 & $1: 44: 46.0$ & 28:03:03.0 & -0.67 & 5.85 & $0.138 \pm 0.026$ & $20.42 \pm 0.05$ \\
\hline 57 & $1: 47: 12.8$ & 28:01:37.7 & -1.66 & 5.20 & $0.129 \pm 0.020$ & $21.76 \pm 0.20$ \\
\hline 58 & $2: 23: 33.2$ & $25: 32: 42.6$ & -0.42 & 5.85 & $0.159 \pm 0.031$ & $19.23 \pm 0.08$ \\
\hline 59 & $2: 24: 01.6$ & $25: 28: 17.3$ & -1.62 & 5.41 & $0.163 \pm 0.030$ & $21.12 \pm 0.33$ \\
\hline 60 & $2: 28: 33.7$ & $25: 19: 51.1$ & -0.53 & 5.80 & $0.186 \pm 0.031$ & $17.66 \pm 0.03$ \\
\hline 61 & 2:31:01.6 & $26: 33: 23.4$ & -1.38 & 4.52 & $0.073 \pm 0.023$ & $22.05 \pm 0.20$ \\
\hline
\end{tabular}




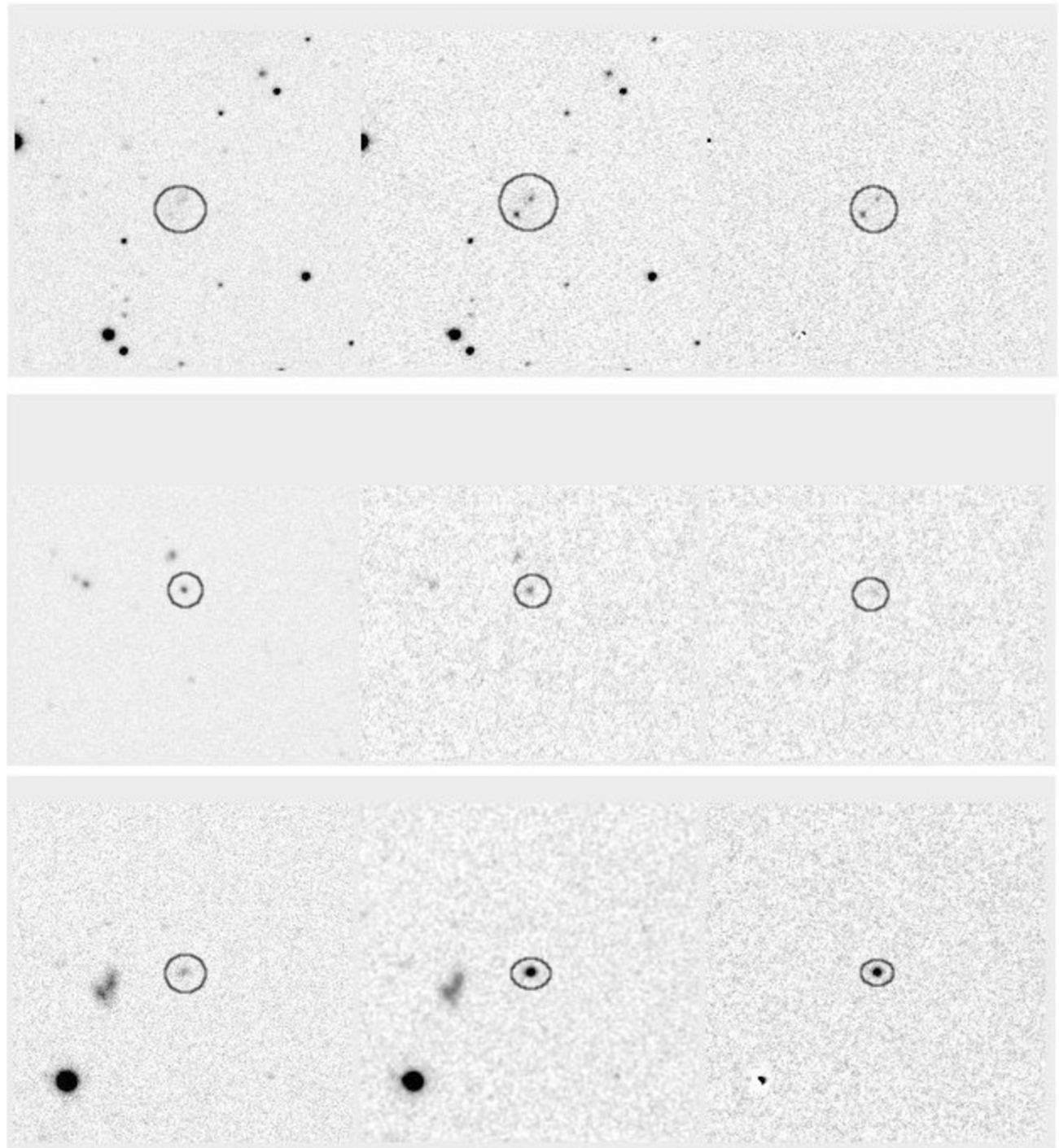

Figure 3. Example $\mathrm{H} \alpha$ dots. Each panel consists of three $200 \times 200$ pixel cutouts $(120 \times 120 \operatorname{arcsec})$ centered on the putative $\mathrm{H} \alpha$ dot. From left to right are the $R$, $\mathrm{H} \alpha$, and continuum-subtracted $\mathrm{H} \alpha$ images. The top panel shows $\mathrm{H} \alpha$ dots 39 and 40 (two $\mathrm{H} \alpha$ dots that are in close proximity), the middle panel shows $\mathrm{H} \alpha$ dot 27 , and the lower set of images represents $\mathrm{H} \alpha$ dot 31 . Notice that the various $\mathrm{H} \alpha$ dots are all relatively faint in the broadband image, but have a wide range of brightnesses in the narrow band.

(epoch J2000), magnitude difference $\Delta m$ and ratio as described in the previous section, the measured emission-line flux and its associated error as measured from the continuum-subtracted narrowband images, and the measured broadband $R$ magnitude (in Vega magnitudes) and its associated error.

A histogram of the emission-line fluxes of the $\mathrm{H} \alpha$ dots is shown in Figure 4. Since the dots are detected via the strength of their emission lines, this plot shows the effective sensitivity of the survey method. As will be discussed in the next section, some of the $\mathrm{H} \alpha$ dots are higher-redshift background objects. For these sources, the value listed in the emission-line flux column of Table 2 does not actually correspond to the $\mathrm{H} \alpha$ flux, but instead is a measurement of the flux of another emission line, such as $[\mathrm{O} \mathrm{III}] \lambda 5007$, that was redshifted into the $\mathrm{H} \alpha$ filter. What is tabulated and plotted in Figure 4 is the flux of the emission line that is present in the narrowband filter. In the case of $\mathrm{H} \alpha$, some of the flux is contributed by the $[\mathrm{N}$ II] $\lambda \lambda 6548,6583$ doublet. While we can correct for the [N II] flux contamination (see subsequent sections), what is presented in the table and plotted in Figure 4 represents the observed flux measured in the narrowband image. The median-detected line flux from the $\mathrm{H} \alpha$ dots is $2.6 \times 10^{-15} \mathrm{erg} \mathrm{s}^{-1} \mathrm{~cm}^{-2}$. For comparison, the median flux from the ALFALFA $\mathrm{H} \alpha$ project galaxies in the same fields is $3.2 \times 10^{-14} \mathrm{erg} \mathrm{s}^{-1} \mathrm{~cm}^{-2}$. As expected, the $\mathrm{H} \alpha$ dots reach down to much lower flux limits than the ALFALFA $\mathrm{H} \alpha$ galaxies. The ability of the $\mathrm{H} \alpha$ dot survey to detect objects with such low flux levels (as low as $6.1 \times 10^{-16} \mathrm{erg} \mathrm{s}^{-1} \mathrm{~cm}^{-2}$ ) suggests that the narrowband imaging technique and $\mathrm{H} \alpha$ dot software are very sensitive.

Figure 5 shows a histogram of the calibrated $R$ magnitudes of the $\mathrm{H} \alpha$ dots. The $R$ magnitudes of the $\mathrm{H} \alpha$ dots range from 15.83 to 22.64 , with a median of 19.49 . As with Figure 4, all objects in Table 2 are included in this plot, although the duplicate object only appears once. The extreme faintness of a number of the $\mathrm{H} \alpha$ dots is another indicator of the sensitivity of this selection method. Furthermore, it suggests that many of the $\mathrm{H} \alpha$ dots may be extremely-low-luminosity systems. Objects detected via $\mathrm{H} \alpha$ emission will be located at relatively small distances (between 20 and $110 \mathrm{Mpc}$ for $H_{0}=70 \mathrm{~km} \mathrm{~s}^{-1} \mathrm{Mpc}^{-1}$ ). Assuming a characteristic distance of $50 \mathrm{Mpc}$, an $\mathrm{H} \alpha$ dot with the median $R$ magnitude of the sample will have an absolute magnitude $M_{R}=-14.0$ (i.e., several times less luminous than 


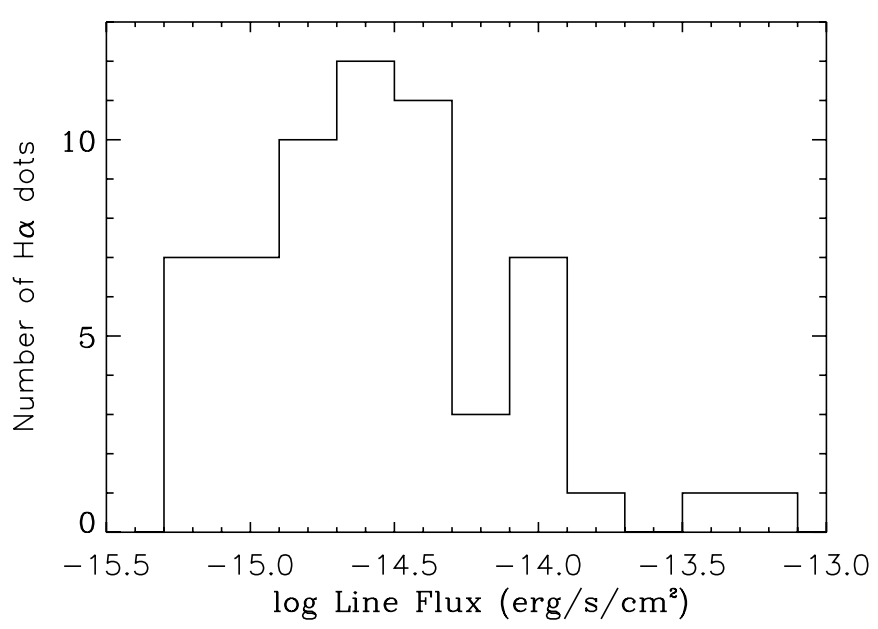

Figure 4. Histogram of the $\mathrm{H} \alpha$ dot emission-line fluxes measured from the narrowband images (in erg s $\mathrm{s}^{-1} \mathrm{~cm}^{-2}$ ). The logarithm of the median line flux is -14.58 .

the Small Magellanic Cloud, SMC). It should be clear that many very-low-luminosity star-forming dwarfs will be found among the $\mathrm{H} \alpha$ dots.

We note in passing that the one object that was detected in two separate fields, $\mathrm{H} \alpha \operatorname{dot} 7=\mathrm{H} \alpha$ dot 24 , is also the faintest object in the sample, with $R=22.6$. It also has one of the lowest line fluxes in the overall sample. While it is strictly a coincidence that the single duplicate $\mathrm{H} \alpha$ dot is also the faintest, this fact does speak to the robustness and repeatability of the survey method. Despite the extremely low flux levels from this source, it was detected twice on overlapping images taken on separate observing runs.

\section{SPECTROSCOPIC FOLLOW-UP}

\subsection{The Need for Spectroscopic Follow-up}

Having identified a large sample of $\mathrm{H} \alpha$ dot candidates, we needed spectra in order to determine their nature and measure their physical properties. Using a wide-field CCD and narrowband imaging is an excellent way to identify emissionline objects. However, there are inherent ambiguities in this type of survey. The specific wavelength range of the $\mathrm{H} \alpha$ filters was chosen to correspond to the distance of the target ALFALFA galaxy in the field so that the $\mathrm{H} \alpha$ flux and starformation properties of the target galaxy could be measured. Assuming that the $\mathrm{H} \alpha$ dots are at a redshift similar to the target ALFALFA galaxy, the excess emission-line flux is indicative of $\mathrm{H} \alpha$ emission. The narrowband filters can detect the $\mathrm{H} \alpha$ emission line of an $\mathrm{H} \alpha$ dot over a velocity range from 0 to $7810 \mathrm{~km} \mathrm{~s}^{-1}$ (see Table 1). However, the $\mathrm{H} \alpha$ dot is not necessarily at a redshift similar to the target ALFALFA galaxy. It could be an emissionline object at a higher redshift, such that another strong emission line such as [O III] $\lambda 5007$ is redshifted into the $\mathrm{H} \alpha$ filter and shows up as a detection. Based on the wavelength range of the filters, we expect that the [O III]-detected objects will lie in a redshift range from $z=0.311-0.345$. It is also possible that the $\mathrm{H} \alpha$ dots could be quasars, in which one of the prominent quasar emission lines (e.g., White et al. 2000) is redshifted into the $\mathrm{H} \alpha$ filter. It is necessary to obtain spectra of the $\mathrm{H} \alpha$ dots in order to determine their redshift and ascertain which emission line is present in the narrowband filter.

Follow-up spectra also allow us to distinguish between star-forming objects and AGNs. Observationally, star-forming

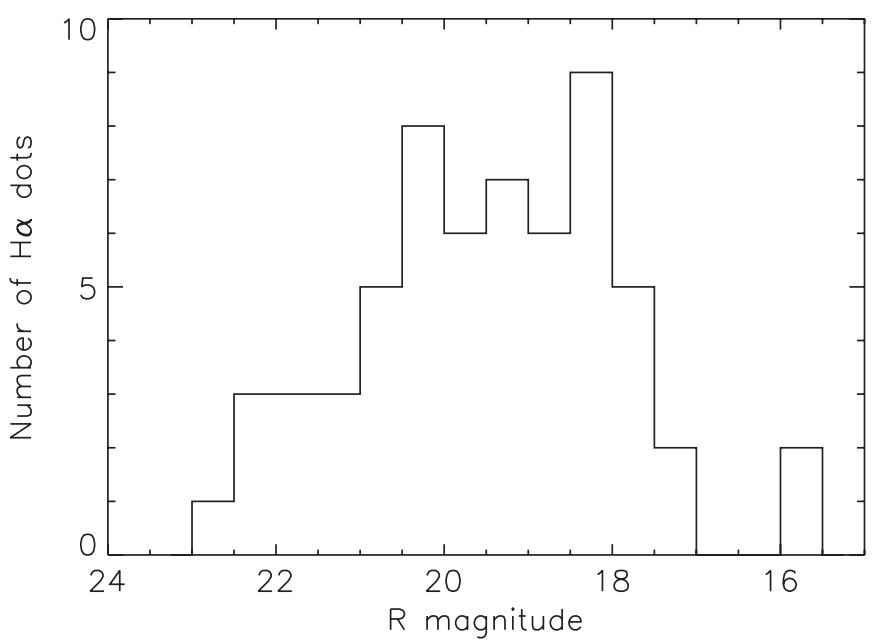

Figure 5. Histogram of the $R$-band magnitudes of the $\mathrm{H} \alpha$ dots. The median value is 19.49 , and sources as faint as 22.6 are included in the survey.

galaxies can be distinguished from AGNs based on the relative strengths of their emission lines. Emission-line diagnostics such as the $\left[\mathrm{N}_{\mathrm{II}}\right] \lambda 6583 / \mathrm{H} \alpha$ ratio and the $\left[\mathrm{O}_{\mathrm{III}}\right] \lambda 5007 / \mathrm{H} \beta$ ratio can be used to determine the activity type of each object.

Another key benefit of obtaining follow-up spectroscopy is that the data can be used to estimate metal abundances for the sources where the emission is dominated by star-formation processes. There are three important applications for the $\mathrm{H} \alpha$ dot sample in this regard. As we implied earlier, for at least some of the $\mathrm{H} \alpha$-selected dots these objects will be among the lowest luminosity actively star-forming systems in the local universe. Obtaining metallicities for these objects will be very important, since low-luminosity galaxies tend to have very low metallicities. Second, some of these lower-redshift $\mathrm{H} \alpha$ selected objects may be isolated extragalactic $\mathrm{H}_{\text {II }}$ regions of the type studied by Ryan-Weber et al. (2004) and Werk et al. (2008, 2010). Any dot with an unusually high metal abundance for its luminosity may well be an isolated $\mathrm{H}$ II region created from tidally stripped, metal-enriched gas from a nearby galaxy. Hence, the measurement of its abundance could help to classify it definitively as a isolated extragalactic $\mathrm{H}$ II region rather than an ultra-low-luminosity dwarf galaxy. Third, for star-forming galaxies detected via their [O III] $\lambda 5007$ emission, there is a strong possibility that the object is a low abundance $\sim L^{*}$ galaxy of the type described by Salzer et al. (2009). The discovery of additional examples of this enigmatic class of galaxy would be particularly interesting.

\subsection{Spectral Observations and Data Reduction}

\subsubsection{MDM Spectral Observations}

Spectra of most of the $\mathrm{H} \alpha$ dot candidates presented in Table 2 were obtained using the $\mathrm{MDM}^{8}$ Hiltner $2.4 \mathrm{~m}$ telescope in 2007 November and 2008 March. The spectra were acquired using the CCDS spectrograph and a low dispersion grating (150 lines $\mathrm{mm}^{-1}$ ) that allowed us to cover the entire optical spectrum from $3720 \AA$ to $7300 \AA$, centered at $5400 \AA$. The reciprocal dispersion of this spectrograph setup is $3.066 \AA$ pixel $^{-1}$. The slit angle was aligned east-west, which was close to the parallactic angle for nearly all of the observations. Each object was observed

\footnotetext{
8 Based on observations obtained at the MDM Observatory, which is a operated by a consortium consisting of the University of Michigan, Dartmouth College, Ohio State University, Columbia University, and Ohio University.
} 
for at least two separate 20 minute integrations. Additional integrations were taken for candidates that appeared to have strong emission lines. Since many of the $\mathrm{H} \alpha$ dot candidates are very faint, we could not always see the object in the slit and therefore had to do blind offsets from nearby bright stars. Calibration images, including internal flat lamps, twilight flats, $\mathrm{Ne}$ and $\mathrm{Hg}-\mathrm{Ne}$ comparison lamps, and biases were taken. We also observed spectrophotometric standard stars (e.g., Oke \& Gunn 1983; Massey et al. 1988) throughout each night.

The spectra were reduced using IRAF, and standard reduction steps were followed. The bias level was removed using the overscan section present in each image. We corrected for pixel-to-pixel sensitivity variations by dividing by an averagecombined internal flat lamp image. Cosmic rays were removed from the object spectra using the program LACOS_SPEC (van Dokkum 2001). One-dimensional spectra were extracted from the two-dimensional images, after which the spectra were wavelength calibrated using the $\mathrm{Ne}$ and $\mathrm{Hg}-\mathrm{Ne}$ comparison lamp spectra, and flux calibrated using a sensitivity function derived using the standard star observations.

\subsubsection{HET Spectral Observations}

Additional spectra for $\mathrm{H} \alpha$ dot candidates were obtained with the 9.2 m Hobby-Eberly Telescope ${ }^{9}$ (HET) between 2008 August and 2011 January. The HET observations fall into two categories. Several $\mathrm{H} \alpha$ dots were not observed during our initial MDM sessions. These were mainly objects located in the Fall survey fields. In addition, we required spectra in the far red $(\sim 9000 \AA)$ in order to observe the $\mathrm{H} \alpha$ spectral region for the higher-redshift [O III]-detected $\mathrm{H} \alpha$ dots. These red spectra were necessary for object classification, since the $[\mathrm{N} \mathrm{II}] / \mathrm{H} \alpha$ and $[\mathrm{S} \mathrm{II}] / \mathrm{H} \alpha$ ratios are useful for distinguishing between starforming galaxies and narrow-line AGNs.

All observations were acquired using the Marcario Low Resolution Spectrograph (LRS; Hill et al. 1998) and either the $\mathrm{G} 2$ or G3 gratings. The LRS-plus-G2 combination provides a spectral coverage between $4350 \AA$ and $7250 \AA$ with a reciprocal dispersion of $2.00 \AA$ pixel $^{-1}$. This spectral setting was used for all "first-look" spectra. We observed $11 \mathrm{H} \alpha$ dots in the Fall 2008 semester in this mode. For objects observed at MDM that had been discovered via their [O III] emission (redshifts of $0.317-0.343$ ), we used grating G3 to observe the wavelength range 6300-9100 $\AA$ at $1.91 \AA$ pixel $^{-1}$. This allowed us to detect both $\mathrm{H} \alpha$ and the $[\mathrm{N} \mathrm{II}] \lambda \lambda 6568,6583$ doublet for these objects. In several of these sources we also detected [S II] $\lambda \lambda 6717,6731$ as well. A total of 17 [O III]-detected objects were observed using G3.

Processing of the HET spectra followed a procedure similar to the one employed for the MDM data. All images were bias corrected using overscan sections and mean bias images created using typically seven zero-exposure images provided each night by the HET queue observers. Several flat-field images, also taken nightly, were combined into a single frame, normalized, and applied to the science images. Cosmic rays were rejected using LACOS_SPEC (van Dokkum 2001), after which one-dimensional spectra were extracted from the images. Wavelength calibrations were provided using spectra of $\mathrm{Ne}$ and $\mathrm{Cd}$ lamps, and a single standard star observed nightly provided the relative flux scale.

\footnotetext{
9 Based on observations obtained with the Hobby-Eberly Telescope, which is a joint project of the University of Texas at Austin, the Pennsylvania State University, Stanford University, Ludwig-Maximilians-Universität München, and Georg-August-Universität Göttingen.
}

\subsection{Results of Spectroscopy}

Spectra for all $60 \mathrm{H} \alpha$ dot candidates were obtained as described above. Example spectra of several objects are shown in Figures 6-8. Once the spectral reductions were completed, spectral features were measured in the reduced spectra using the IRAF SPLOT routine. The resulting measured emission-line wavelengths were used to determine the redshift of each $\mathrm{H} \alpha$ dot. The emission-line fluxes were corrected for reddening using the observed Balmer line ratios $(\mathrm{H} \alpha / \mathrm{H} \beta)$.

Relevant spectral data are summarized in Table 3. The $\mathrm{H} \alpha$ dot number is given in Column 1, followed by the ELG type. This designation has the following meaning: SF: star-forming galaxy (regardless of redshift); Sy1: Seyfert 1 galaxy; Sy2: Seyfert 2 galaxy; LINER: low-ionization nuclear emission region; QSO: quasi-stellar object/quasar; Not ELG: spectrum does not include any detectable emission features. This latter class represents false detections in our initial list of $\mathrm{H} \alpha$ dot candidates. These false detections can only be recognized as such after a spectrum has been acquired. Column 3 lists the spectroscopic redshift, derived from the average redshifts of all strong emission lines. Typical formal uncertainties for the redshifts, based on (1) the measurement uncertainties of individual lines, (2) the formal error from the wavelength solution, and (3) the scatter in the determination of the mean redshift from the multiple lines, are $15-30 \mathrm{~km} \mathrm{~s}^{-1}$. Column 4 lists the decimal reddening coefficient $c_{\mathrm{H} \beta}$, derived from the Balmer-line ratio $\mathrm{H} \alpha / \mathrm{H} \beta$ (Osterbrock 1989). The equivalent widths of the $\mathrm{H} \alpha$ and [O III] lines are listed in Columns 5 and 6 . Key reddening-corrected emission-line ratios are given in Columns 7-10: $\log \left(\left[\mathrm{N}_{\mathrm{II}}\right] \lambda 6583 / \mathrm{H} \alpha\right), \log ([\mathrm{O}$ III $] \lambda 5007 /$ $\mathrm{H} \beta), \log ([\mathrm{O} \mathrm{II}] \lambda 3727 / \mathrm{H} \beta)$, and $\log ([\mathrm{S} \mathrm{II}] \lambda \lambda 6717,6731 / \mathrm{H} \alpha)$. Objects where one or more of these line ratios are missing represent targets where the relevant lines were either not measurable or (most often) redshifted out of the observed spectral region. Line ratios flagged by an asterisk (*) indicate objects for which $c_{\mathrm{H} \beta}$ could not be measured or was deemed unreliable. For these objects the emission-line ratios listed are not reddening corrected. Finally, Column 11 lists an estimate of the metallicity of the system for the star-forming objects. The derivation of the abundances is described in detail in Section 6.2.

\subsubsection{Redshifts and ELG Types}

Our follow-up spectra allow us to measure the redshift of each $\mathrm{H} \alpha$ dot as well as to ascertain the emission line that was present in the narrowband imaging data. The $\mathrm{H} \alpha$ dots can be separated into four primary categories. These are (1) $\mathrm{H} \alpha$ detected sources, whose redshifts are relatively close to those of the ALFALFA $\mathrm{H} \alpha$ targets; (2) [O III]-detected galaxies that lie in the redshift range $0.311-0.345$; (3) QSOs detected by one of several possible emission lines, and (4) non-ELGs, objects with no visible emission lines that are false detections. We discuss each of these groups in turn.

$H \alpha$-detections. Of the 60 unique $\mathrm{H} \alpha$ dots in our sample, 29 of them $(48 \%)$ are low redshift and lie in the range $z=0.006$ to $z=0.024$. Figure 6 shows example spectra of three low-redshift $\mathrm{H} \alpha$ dots, all obtained at MDM. For these objects, the emissionline detected in the narrowband images is in fact $\mathrm{H} \alpha$; hence the $\mathrm{H} \alpha$ emission line is prominent in each spectrum. These spectra are typical of low-luminosity, low-metallicity objects with [O III] $\lambda 5007 / \mathrm{H} \beta$ ratios greater than $\sim 3$ and $\left[\mathrm{N}_{\text {II }}\right] \lambda 6583 /$ $\mathrm{H} \alpha$ ratios less than 0.1 . Other emission lines present in these three spectra include [O II] $\lambda 3727, \mathrm{H} \gamma$, and [S II] $\lambda \lambda 6717,6731$. 
Table 3

Spectroscopic Results

\begin{tabular}{|c|c|c|c|c|c|c|c|c|c|c|}
\hline $\begin{array}{l}\mathrm{H} \alpha \text { Dot } \\
\text { (1) }\end{array}$ & $\begin{array}{l}\text { ELG Type } \\
\text { (2) }\end{array}$ & $\begin{array}{l}z \\
(3)\end{array}$ & $\begin{array}{l}c_{\mathrm{H} \beta} \\
(4)\end{array}$ & $\begin{array}{c}\text { EW H } \alpha \\
(\AA) \\
(5)\end{array}$ & $\begin{array}{c}\text { EW [O III] } \\
(\AA) \\
(6)\end{array}$ & $\begin{array}{c}{[\mathrm{N} \text { II }] / \mathrm{H} \alpha} \\
(7)\end{array}$ & $\begin{array}{c}{[\mathrm{O} \mathrm{III}] / \mathrm{H} \beta} \\
(8)\end{array}$ & $\begin{array}{c}{[\mathrm{O} \mathrm{II}] / \mathrm{H} \beta} \\
\text { (9) }\end{array}$ & $\begin{array}{c}{[\mathrm{S} \text { II }] / \mathrm{H} \alpha} \\
\quad(10)\end{array}$ & $\begin{array}{c}\log [\mathrm{O} / \mathrm{H}]+12 \\
(11)\end{array}$ \\
\hline 1 & Not ELG & & $\ldots$ & $\ldots$ & $\ldots$ & $\ldots$ & $\ldots$ & . & $\ldots$ & $\ldots$ \\
\hline 2 & $\mathrm{SF}$ & 0.0109 & 0.21 & 154.6 & 114.9 & -1.75 & 0.53 & 0.21 & -1.08 & 7.79 \\
\hline 3 & SF & 0.0110 & 0.11 & 172.2 & 90.9 & -1.43 & 0.34 & 0.09 & -0.84 & 7.96 \\
\hline 4 & SF & 0.0113 & 0.24 & 410.7 & 431.7 & -1.81 & 0.71 & 0.13 & -1.14 & 7.77 \\
\hline 5 & Sy2 & 0.3221 & $\ldots$ & $\ldots$ & 61.0 & $-0.20 *$ & $1.13 *$ & $0.16^{*}$ & $\ldots$ & $\ldots$ \\
\hline 6 & SF & 0.3245 & 0.82 & 70.7 & 37.0 & -1.41 & 0.41 & 0.58 & $\ldots$ & 7.97 \\
\hline 7 & SF & 0.3216 & $\ldots$ & $\ldots$ & 492.1 & $\ldots$ & $0.68^{*}$ & & $\ldots$ & $\ldots$ \\
\hline 8 & SF & 0.0117 & 0.24 & 99.8 & 35.4 & $-1.08^{*}$ & $0.35^{*}$ & $0.47 *$ & $\ldots$ & $\ldots$ \\
\hline 9 & Not ELG & $\ldots$ & $\ldots$ & $\ldots$ & $\ldots$ & $\ldots$ & $\ldots$ & $\ldots$ & $\ldots$ & $\ldots$ \\
\hline 10 & SF & 0.0162 & -0.10 & 73.6 & 74.6 & -1.80 & 0.61 & 0.14 & -1.02 & 7.77 \\
\hline 11 & SF & 0.0092 & $\ldots$ & 297.0 & 79.8 & $-0.92^{*}$ & $\ldots$ & $\ldots$ & $\ldots$ & $\ldots$ \\
\hline 12 & SF & 0.0059 & 0.08 & 156.9 & 143.4 & -1.71 & 0.62 & 0.13 & -1.05 & 7.81 \\
\hline 13 & SF & 0.0066 & 0.19 & 56.2 & 15.2 & -1.89 & 0.06 & 0.42 & -0.77 & 7.73 \\
\hline 14 & Sy1.9 & 0.3234 & 0.84 & 59.1 & 165.3 & $-0.48^{*}$ & $1.01^{*}$ & $\ldots$ & $\ldots$ & $\ldots$ \\
\hline 15 & SF & 0.3352 & 1.18 & 135.3 & 74.8 & -1.00 & 0.42 & 0.61 & $\ldots$ & 8.31 \\
\hline 16 & SF & 0.0173 & 0.26 & 101.2 & 78.0 & -1.44 & 0.55 & 0.54 & -0.86 & 7.96 \\
\hline 17 & QSO & 1.3472 & $\ldots$ & $\ldots$ & $\ldots$ & $\ldots$ & $\ldots$ & $\ldots$ & $\ldots$ & $\ldots$ \\
\hline 18 & $\mathrm{SF}$ & 0.0121 & 0.27 & 83.3 & 67.8 & -1.26 & 0.57 & $\ldots$ & -0.73 & 8.07 \\
\hline 19 & SF & 0.3208 & 0.29 & 393.3 & 481.5 & -1.96 & 0.69 & 0.30 & $\ldots$ & 7.70 \\
\hline 20 & SF & 0.0147 & 0.52 & 211.2 & 131.5 & -1.59 & 0.46 & 0.35 & -0.89 & 7.87 \\
\hline 21 & Sy2 & 0.3227 & 1.23 & 86.0 & 251.3 & $-0.16^{*}$ & $1.26^{*}$ & $0.54 *$ & $\ldots$ & $\ldots$ \\
\hline 22 & SF & 0.3289 & 0.75 & $\ldots$ & 216.2 & $\ldots$ & $0.54 *$ & $0.48^{*}$ & $\ldots$ & $\ldots$ \\
\hline 23 & Not ELG & $\ldots$ & $\ldots$ & $\ldots$ & $\ldots$ & $\ldots$ & $\ldots$ & $\ldots$ & $\ldots$ & $\ldots$ \\
\hline 24 & SF & 0.3216 & $\ldots$ & $\ldots$ & 492.1 & $\ldots$ & $0.68^{*}$ & $\ldots$ & $\ldots$ & $\ldots$ \\
\hline 25 & Not ELG & $\ldots$ & $\ldots$ & $\ldots$ & $\ldots$ & $\ldots$ & $\ldots$ & $\ldots$ & $\ldots$ & $\ldots$ \\
\hline 26 & Not ELG & $\ldots$ & $\ldots$ & $\ldots$ & $\ldots$ & $\ldots$ & $\ldots$ & $\ldots$ & $\ldots$ & $\ldots$ \\
\hline 27 & QSO & 3.2853 & $\ldots$ & $\ldots$ & $\ldots$ & $\ldots$ & $\ldots$ & $\ldots$ & $\ldots$ & $\ldots$ \\
\hline 28 & $\mathrm{SF}$ & 0.3217 & $\ldots$ & $\ldots$ & 612.8 & $-1.50 *$ & $0.82 *$ & $0.21 *$ & $\ldots$ & $\ldots$ \\
\hline 29 & Not ELG & $\ldots$ & $\ldots$ & $\ldots$ & $\ldots$ & $\ldots$ & $\ldots$ & $\ldots$ & $\ldots$ & $\ldots$ \\
\hline 30 & Sy2/LINER & 0.3173 & $\ldots$ & $\ldots$ & 75.6 & $-0.40 *$ & $0.41 *$ & $0.63 *$ & $\ldots$ & $\ldots$ \\
\hline 31 & SF & 0.0151 & 0.01 & 409.8 & 483.6 & -1.89 & 0.72 & 0.04 & -1.11 & 7.73 \\
\hline 32 & SF & 0.0175 & 0.13 & 106.0 & 74.7 & -1.50 & 0.50 & 0.48 & -0.61 & 7.92 \\
\hline 33 & SF & 0.0139 & 0.45 & 72.7 & 72.1 & -1.46 & 0.61 & 0.61 & -0.83 & 7.95 \\
\hline 34 & SF & 0.0173 & 0.25 & 167.8 & 80.5 & -1.51 & 0.38 & 0.53 & -0.83 & 7.92 \\
\hline 35 & SF & 0.0163 & 0.99 & 22.3 & 10.2 & $-1.17^{*}$ & $0.56^{*}$ & $1.19^{*}$ & $-0.23^{*}$ & $\ldots$ \\
\hline 36 & QSO & 3.2864 & $\ldots$ & $\ldots$ & $\ldots$ & $\ldots$ & $\ldots$ & $\ldots$ & $\ldots$ & $\ldots$ \\
\hline 37 & $\mathrm{SF}$ & 0.3303 & 0.60 & 245.5 & 189.7 & -1.30 & 0.65 & 0.55 & $\ldots$ & 8.04 \\
\hline 38 & Sy2 & 0.3338 & 1.41 & 40.4 & 49.1 & $-0.29^{*}$ & $0.87 *$ & $0.87 *$ & $\ldots$ & $\ldots$ \\
\hline 39 & SF & 0.0168 & 0.47 & 141.0 & 72.9 & -1.78 & 0.39 & 0.40 & -0.90 & 7.78 \\
\hline 40 & SF & 0.0163 & 0.34 & 722.0 & 538.1 & -1.74 & 0.59 & 0.27 & -1.01 & 7.80 \\
\hline 41 & SF & 0.0163 & 0.56 & 91.1 & 50.7 & -0.97 & 0.43 & 0.63 & -0.62 & 8.31 \\
\hline 42 & Sy2 & 0.3313 & $\ldots$ & $\ldots$ & 174.6 & $-0.03^{*}$ & $0.92 *$ & $0.24 *$ & $\ldots$ & $\ldots$ \\
\hline 43 & $\mathrm{SF}$ & 0.0208 & -0.05 & 178.9 & 152.7 & -1.19 & 0.58 & 0.76 & -0.93 & 8.12 \\
\hline 44 & $\mathrm{SF}$ & 0.0240 & 0.72 & 41.1 & 22.5 & -1.19 & 0.44 & 0.68 & $\ldots$ & 8.11 \\
\hline 45 & Not ELG & $\ldots$ & $\ldots$ & $\ldots$ & $\ldots$ & $\ldots$ & $\ldots$ & $\ldots$ & $\ldots$ & $\ldots$ \\
\hline 46 & $\mathrm{SF}$ & 0.0173 & 0.17 & 59.3 & 22.2 & -1.32 & 0.18 & 0.33 & -0.62 & 8.03 \\
\hline 47 & SF & 0.0169 & 0.29 & 990.9 & 716.9 & -1.85 & 0.54 & $\ldots$ & $\ldots$ & 7.75 \\
\hline 48 & SF & 0.0239 & 0.41 & 111.8 & 116.1 & -1.40 & 0.70 & 0.48 & $\ldots$ & 7.98 \\
\hline 49 & SF & 0.0171 & 0.21 & 49.0 & 37.2 & -1.61 & 0.49 & 0.58 & -0.68 & 7.86 \\
\hline 50 & Not ELG & $\ldots$ & $\ldots$ & $\ldots$ & $\ldots$ & $\ldots$ & $\ldots$ & $\ldots$ & $\ldots$ & $\ldots$ \\
\hline 51 & $\mathrm{SF}$ & 0.0170 & 0.60 & 35.3 & 16.4 & -2.17 & 0.35 & $\ldots$ & -0.88 & 7.62 \\
\hline 52 & QSO & 2.5433 & $\ldots$ & $\ldots$ & $\ldots$ & $\ldots$ & $\ldots$ & $\ldots$ & $\ldots$ & $\ldots$ \\
\hline 53 & $\mathrm{SF}$ & 0.0230 & 0.55 & 153.4 & 141.1 & -1.15 & 0.64 & 0.55 & -1.16 & 8.14 \\
\hline 54 & Not ELG & $\ldots$ & $\ldots$ & $\ldots$ & $\ldots$ & $\ldots$ & $\ldots$ & $\ldots$ & $\ldots$ & $\ldots$ \\
\hline 55 & $\mathrm{SF}$ & 0.3387 & 0.24 & 360.9 & 506.0 & -2.05 & 0.74 & 0.16 & $\ldots$ & 7.66 \\
\hline 56 & SF & 0.3394 & 0.66 & 67.5 & 21.4 & -1.45 & 0.15 & 0.88 & $\ldots$ & 7.95 \\
\hline 57 & SF & 0.3395 & -0.09 & 441.8 & 580.8 & -1.91 & 0.87 & 0.12 & $\ldots$ & 7.72 \\
\hline 58 & SF & 0.0179 & 0.03 & 40.8 & 27.4 & -1.42 & 0.39 & 0.47 & -0.67 & 7.97 \\
\hline 59 & SF & 0.3370 & 0.73 & 140.4 & 97.2 & -1.61 & 0.56 & 0.42 & $\ldots$ & 7.86 \\
\hline 60 & H II & 0.0195 & 0.61 & 42.4 & 18.0 & $-0.98^{*}$ & $0.16^{*}$ & $\ldots$ & $-0.46^{*}$ & $\ldots$ \\
\hline 61 & $\mathrm{SF}$ & 0.3428 & 0.25 & 176.6 & 129.3 & -1.40 & 0.55 & 0.53 & $\ldots$ & 7.98 \\
\hline
\end{tabular}




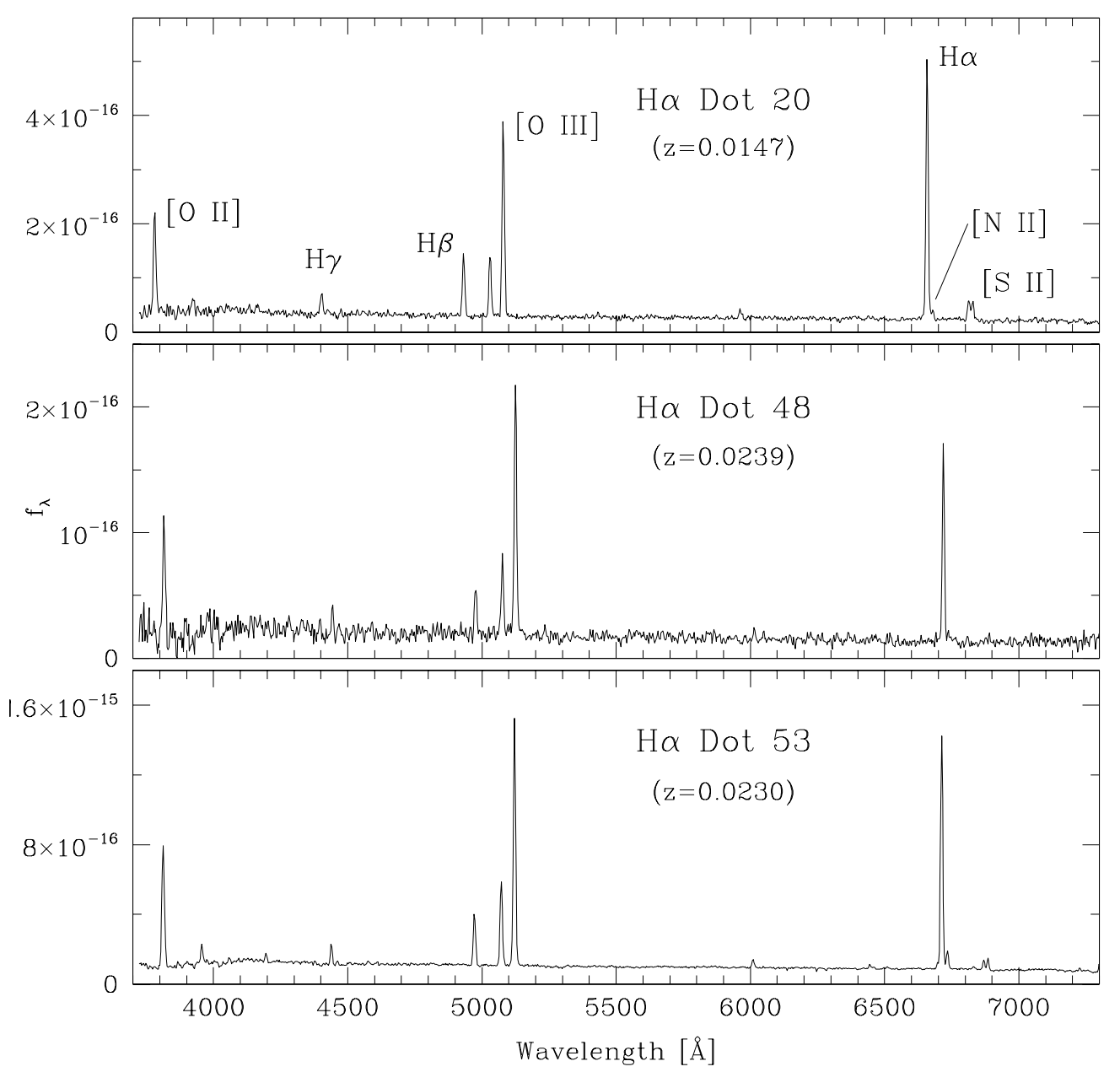

Figure 6. Example spectra of three low-redshift $\mathrm{H} \alpha$ dots taken using the MDM $2.4 \mathrm{~m}$ telescope. All three objects were detected in our survey via their $\mathrm{H} \alpha$ emission line and are star-forming dwarf galaxies. $\mathrm{H} \alpha$ dot 20 (top panel) is the object indicated in the lower portion of the images shown in Figure 1.

All objects in this class are either dwarf star-forming galaxies or $\mathrm{H}$ II regions in the outer regions of other galaxies. The work of Ryan-Weber et al. (2004) and Werk et al. (2010) has raised interest in the detection of so-called isolated extragalactic $\mathrm{H}_{\text {II }}$ regions-H II regions that are located far outside the optical boundaries of larger galaxies. None of the $\mathrm{H} \alpha$-detected objects discussed in the current study obviously qualify as isolated extragalactic H II regions, although one ( $\mathrm{H} \alpha$ dot 60$)$ appears to be a star-forming region on the very outskirts of a dwarf galaxy. There are six criteria that we use when trying to differentiate between extragalactic $\mathrm{H}$ II regions and dwarf starforming galaxies. These are: (1) a very small velocity difference between the $\mathrm{H} \alpha$-detected object and the nearby galaxy; (2) isolated $\mathrm{H}$ II regions look compact in both the $\mathrm{H} \alpha$ continuumsubtracted image and the $R$ image, while star-forming dwarf galaxies appear somewhat diffuse and extended in the $R$ image; however, the object could appear as a point source due to lack of resolution, so this criterion alone is insufficient to identify an object as an extragalactic $\mathrm{H}$ II region; (3) the physical separation between the $\mathrm{H} \alpha$-detected object and the nearest galaxy is small (see Section 6.3); (4) the H $\alpha$-detected object has a high abundance of metals for its luminosity (see Section 6.2); (5) an $\mathrm{H}$ I map indicates a tidal stream of neutral hydrogen connecting the $\mathrm{H} \alpha$-detected object and a nearby galaxy; and (6) the $\mathrm{H} \alpha$ detected object is located in a UV halo surrounding a nearby galaxy, and is connected to the galaxy by a star-forming stream. To explore these latter two criteria, H I maps of some of these
$\mathrm{H} \alpha$-detected objects have been obtained using the Very Large Array (VLA), plus near- and far-UV images of some of the lowredshift $\mathrm{H} \alpha$-detected objects have been obtained using GALEX. These new observations are currently being analyzed and will appear as a separate study. At the moment, we conclude that none of the $\mathrm{H} \alpha$-detected objects are isolated extragalactic $\mathrm{H}_{\text {II }}$ regions, but rather are all star-forming dwarf galaxies (with the exception of $\mathrm{H} \alpha$ dot 60). The $\mathrm{H} \alpha$ dot survey is ongoing, so it is possible that extragalactic $\mathrm{H}$ II regions similar to the objects discovered by Ryan-Weber et al. (2004) and Werk et al. (2010) will be found in subsequent searches.

It is perhaps not too surprising that no isolated extragalactic $\mathrm{H}$ II regions were discovered in our first search for $\mathrm{H} \alpha$ dots, despite the fact that several were located in the studies mentioned above. For one thing, these objects are fairly rare, and hence finding no examples among a sample of a few hundred galaxies (many of which are dwarfs) is not unusual. A second factor, which represents a key difference between our study and those of Ryan-Weber et al. (2004) and Werk et al. (2010), is that the ALFALFA $\mathrm{H} \alpha$ sample is restricted to galaxies with velocities beyond $1500 \mathrm{~km} \mathrm{~s}^{-1}$. The larger average distances of our targets makes it less likely that we could detect the emission from a single isolated $\mathrm{H}$ II region.

[O III]-detections. Twenty-two of the $60 \mathrm{H} \alpha$ dots in our sample $(37 \%)$ are higher-redshift objects. Of these, 18 are objects for which the $\left[\mathrm{O}_{\mathrm{III}}\right] \lambda 5007$ line is redshifted into the narrowband $\mathrm{H} \alpha$ filter. The redshifts of these [O III]-detected 


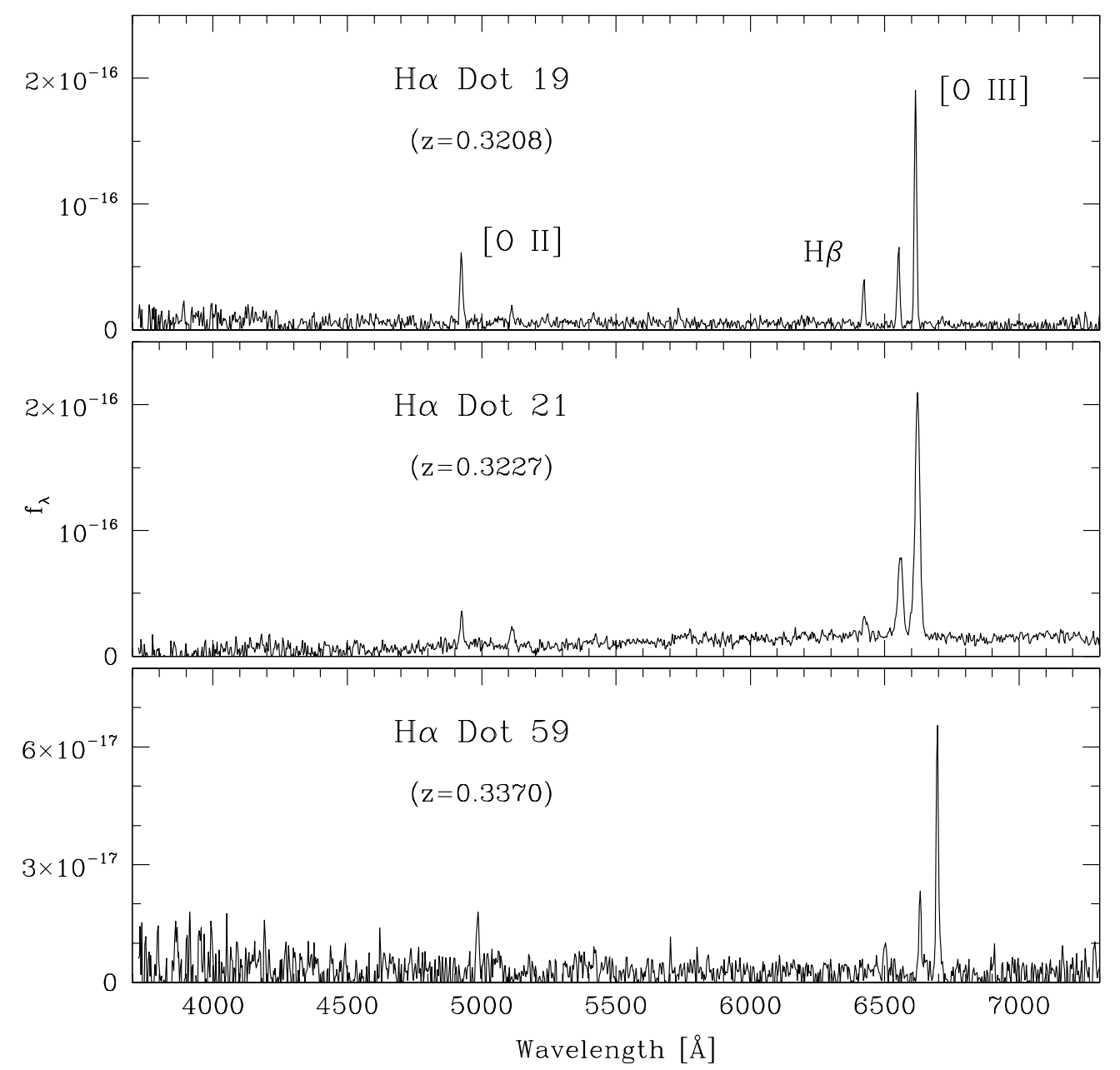

Figure 7. Example spectra of [O III]-detected $\mathrm{H} \alpha$ dots taken using the MDM $2.4 \mathrm{~m}$ telescope. The upper (H $\alpha$ dot 19) and lower (H $\alpha$ dot 59) spectra are of star-forming galaxies, while the middle spectrum ( $\mathrm{H} \alpha \operatorname{dot} 21)$ is a Seyfert 2 galaxy. $\mathrm{H} \alpha$ dot 21 is the object indicated in the upper left portion of the images shown in Figure 1.

galaxies range from $z=0.311$ to $z=0.345$. Figure 7 shows three example spectra of [O III]-detected galaxies. In these cases, the important $\mathrm{H} \alpha$ and $[\mathrm{N}$ II] lines are redshifted beyond the rededge of our spectra. In most cases we have observed the far red portion of the spectrum using the HET and have measured the $[\mathrm{N}$ II] $\lambda 6583 / \mathrm{H} \alpha$ ratios. The combination of the MDM and HET spectra reveals that $\mathrm{H} \alpha$ dot 19 (top spectrum) and $\mathrm{H} \alpha$ dot 59 (bottom spectrum) are star-forming galaxies, while $\mathrm{H} \alpha$ dot 21 is a Seyfert 2 galaxy. Naturally, the [O III]-detected sources are substantially more luminous than the nearby $\mathrm{H} \alpha$-detected galaxies. More details concerning the nature of the higherredshift galaxies appear in subsequent sections.

QSOs. Four of the $\mathrm{H} \alpha$ dots are quasars, for which other strong emission lines are redshifted into the $\mathrm{H} \alpha$ filter. One of the quasars, $\mathrm{H} \alpha$ dot 17 , is at $z=1.347$ and is detected by $\mathrm{Mg}$ II $\lambda 2798$, while $\mathrm{H} \alpha$ dot 52 is detected by [C III] $\lambda 1909$ and has a redshift of $z=2.543$. The remaining two quasars, $\mathrm{H} \alpha$ dot 27 and 36, are detected by their C IV $\lambda 1549$ emission and have redshifts of $z=3.285$ and $z=3.286$, respectively. Figure 8 shows the spectrum for $\mathrm{H} \alpha$ dot 17 , which has a prominent Mg II line redshifted to $6570 \AA$. Weak C III is also visible in the spectrum, helping to confirm the redshift determination.

Non-ELGs. The remaining nine $\mathrm{H} \alpha$ dots (15\% of the sample) are not ELGs. These false detections are caused by two types of contaminants. First, correlated noise pixels in the often noisy
$\mathrm{H} \alpha$ images that happen to correspond exactly with the position of an object in the $R$ image can masquerade as ultra-faint $\mathrm{H} \alpha$ dot candidates. In our early searches, we tended to include objects in our candidate lists even when the putative detection was marginal. In addition, a handful of our false detections turned out to be M stars. Cool M stars have deep absorption troughs, such that their spectra can produce artificial "emission features" between the troughs that mimic emission-line sources. The presence of numerous M-star false detections was noted in the KISS objective-prism survey as well (Salzer et al. 2000, 2001).

The remainder of this paper focuses on the analysis of the 51 real ELGs.

\subsubsection{Distinguishing between Star-forming Galaxies and AGNs}

The emission-line ratios presented in Table 3 can be used to distinguish between galaxies in which the emission is related to star formation and galaxies in which accretion onto a black hole is the dominant source of activity (Baldwin et al. 1981; Veilleux \& Osterbrock 1987; Kewley et al. 2001; Kauffmann et al. 2003). The ELGs are shown in a diagnostic diagram in Figure 9 that plots $\log ([\mathrm{O}$ III $] \lambda 5007 / \mathrm{H} \beta)$ versus $\log ([\mathrm{N}$ II $] \lambda 6583 / \mathrm{H} \alpha)$. The dashed line in the figure is the empirical demarcation from Kauffmann et al. (2003) that divides galaxies with AGN activity from star-forming galaxies. This line, based on a large sample of ELGs from the Sloan Digital Sky Survey, classifies a galaxy 


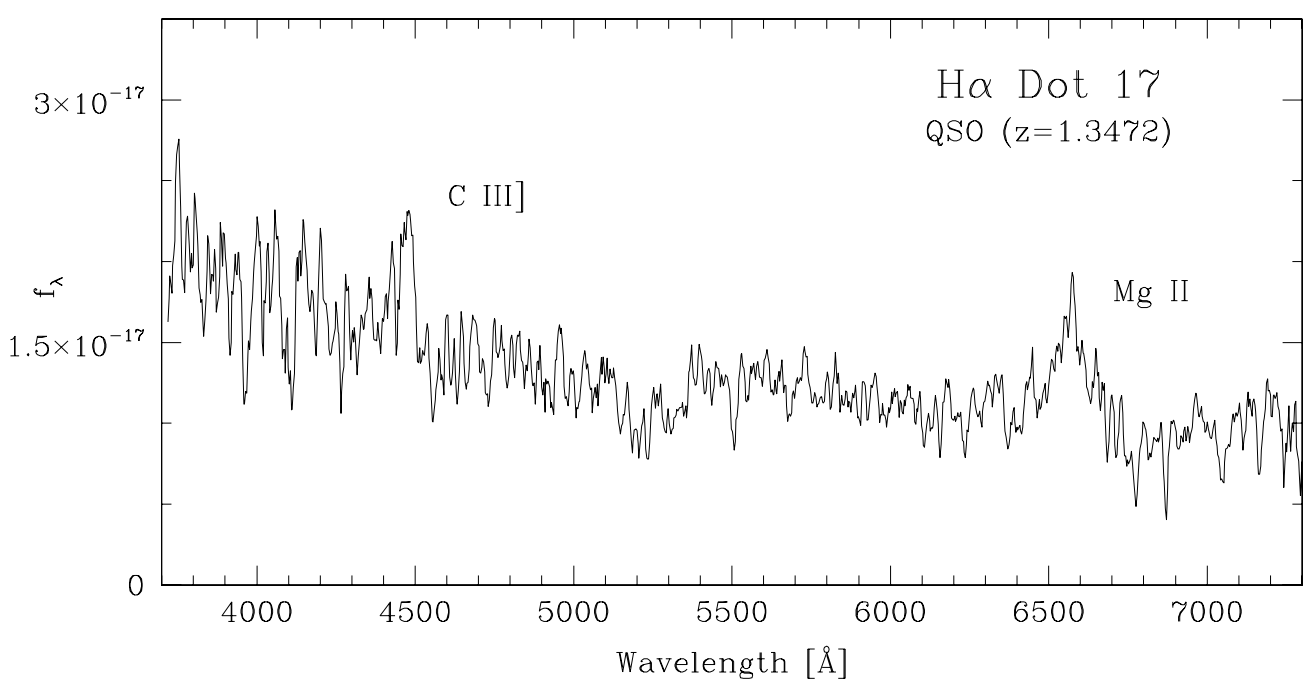

Figure 8. Example spectrum of the quasar $\mathrm{H} \alpha$ dot 17 detected by our survey. This spectrum is taken using the MDM $2.4 \mathrm{~m}$ telescope. The broad line of Mg II is redshifted into the narrowband filter used to detect this object.

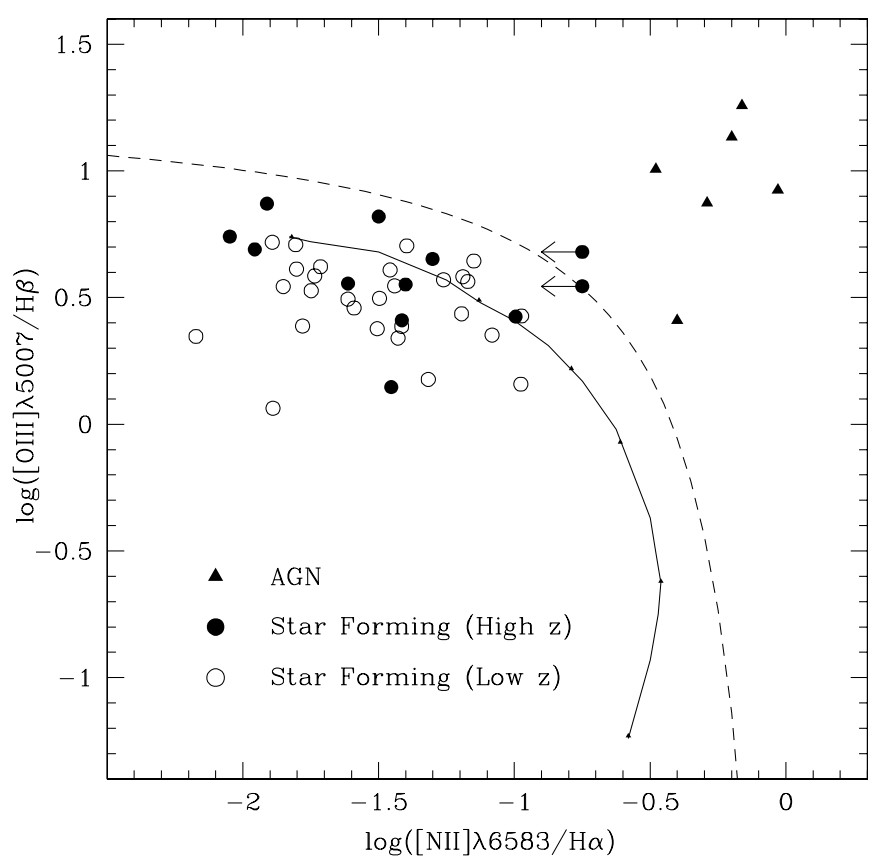

Figure 9. Emission-line ratio diagnostic diagram for the $\mathrm{H} \alpha$ dots. $\mathrm{H} \alpha$-detected (open symbols) vs. [O III]-detected objects (filled symbols) are distinguished from each other, as are star-forming galaxies (circles) vs. AGNs (triangles). The dashed line is from Kauffmann et al. (2003) and delineates regions in the diagram occupied by star-forming galaxies and narrow-line AGNs. The solid line represents a locus of H II region model points from Dopita \& Evans (1986), where the metallicity of the models vary smoothly from high values in the lower right to low values in the upper left.

as an $\mathrm{AGN}$ if

$$
\log ([\mathrm{O} \mathrm{III}] / \mathrm{H} \beta)>0.61 /(\log ([\mathrm{N} \mathrm{II}] / \mathrm{H} \alpha)-0.05)+1.3 .
$$

The solid line represents a locus of nebular models from Dopita \& Evans (1986). The group of models shown here are ones with high-ionization parameters. The metallicity of the models varies smoothly from the lower right (super-solar abundances) to the upper left (sub-solar abundances).

All objects with the necessary emission lines in their observed spectra are plotted in Figure 9. This includes both the
$\mathrm{H} \alpha$-detected (open symbols) and [O III]-detected (closed symbols) objects but excludes the four QSOs. For two of the highredshift galaxies ( $\mathrm{H} \alpha$ dots 7 and 22) we have not yet measured their $[\mathrm{N}$ II $] / \mathrm{H} \alpha$ ratios. In Figure 9, these two galaxies are plotted with a default $[\mathrm{N}$ II $] / \mathrm{H} \alpha$ ratio of -0.75 . This is just where we place them in the diagram and it is not intended to be an upper limit. It is likely, but not ensured, that these two galaxies (indicated by left pointing arrows in the diagram) are star-forming regions based on their $[\mathrm{O} \mathrm{III}] / \mathrm{H} \beta$ ratios. We plan to take additional spectra in order to confirm their activity types. $\mathrm{H} \alpha$ dot 11 is not plotted on the diagnostic diagram because of the poor quality of its spectrum ( $\mathrm{H} \beta$ was not detected). However, we think that it is a star-forming galaxy due to the small observed $[\mathrm{N} \mathrm{II}] / \mathrm{H} \alpha$ ratio.

The star-forming galaxies (circles) lie along the theoretical sequence of $\mathrm{H}$ II regions in Figure 9, and to the left of the Kauffmann dividing line. Interestingly, the [O III]-detected starforming galaxies occupy the same region of the diagnostic diagram as the lower-redshift systems. Both groups appear to be made up of lower-metal-abundance galaxies. As one might expect based on luminosity arguments (Section 4), all 29 of the lower-redshift $\mathrm{H} \alpha$-detected objects are star forming. Additionally, 12 of the 18 [O III]-detected galaxies are also found in this category. The AGNs (filled triangles) lie to the right of the Kauffmann dividing line. All are higher-redshift [O III]-detected systems. Most have high-excitation spectra typical of Seyfert 2 galaxies (large [O III] $/ \mathrm{H} \beta$ ratios). The red HET spectrum of $\mathrm{H} \alpha$ dot 14 reveals weak broad $\mathrm{H} \alpha$ emission, so it has been classified as a Seyfert 1.9. However, we still plot it in Figure 9 (the leftmost of the five high-excitation Seyferts), since we could easily isolate the narrow-line fluxes in this case. The one exception to the highexcitation rule is $\mathrm{H} \alpha$ dot 30, which is located near the Seyfert 2/LINER division in the diagnostic diagram $(\log ([\mathrm{O} \mathrm{III}] / \mathrm{H} \beta) \sim$ $0.5)$. One would normally not expect to detect LINERs via their [O III] emission in a narrowband imaging survey such as ours. The fact that we were able to detect $\mathrm{H} \alpha$ dot 30 speaks to the sensitivity of our data. With the exception of $\mathrm{H} \alpha$ dot 30, the higher-redshift AGNs in the $\mathrm{H} \alpha$ dot sample are located in the same region of the diagnostic diagram as the [O III]-detected KISS Seyfert 2 galaxies detected by Salzer et al. (2009). 


\section{DISCUSSION}

The objects discovered in the $\mathrm{H} \alpha$ dot survey constitute an unusual and diverse population of objects. Selecting objects by their strong line emission pre-selects for high levels of activity - both AGN and star-formation activity. Additionally, the velocity limits set by the narrowband filters used for the ALFALFA $\mathrm{H} \alpha$ project further focuses our sample of emissionline objects. For example, the $\mathrm{H} \alpha$-selected objects are guaranteed to be extremely low luminosity systems; this selection method is apt to detect some of the lowest luminosity star-forming galaxies in the local universe. Correspondingly, the [O III]-detected galaxies must all be fairly luminous systems given the redshift range that this method can detect them over.

Studying the properties of the $\mathrm{H} \alpha$ dots can add to our current understanding of the formation and evolution of galaxies. While the current sample of cataloged emission-line objects is small, we expect the number of objects to grow significantly as the ALFALFA $\mathrm{H} \alpha$ project progresses. Eventually, we expect to have of the order of 300-400 $\mathrm{H} \alpha$ dots cataloged. With such a large number, we expect to be able to apply this sample to address a number of interesting issues. For example, the sample of low-luminosity starbursting galaxies will be large and robust. As noted by Lee et al. (2009), the number of starbursting dwarfs in the very local universe (i.e., within $11 \mathrm{Mpc}$ ) is quite small. Our selection method picks out these objects at much greater distances, but still has the sensitivity to detect very low luminosity star-forming dwarfs. Hence, the dwarf galaxies detected by the $\mathrm{H} \alpha$ dot survey have the potential to complement the very local, volume-limited samples. Specific questions that one can address using the $\mathrm{H} \alpha$-detected objects include probing the nature of starbursts in dwarf galaxies, looking for linkages between the environment of dwarf galaxies and their star-formation characteristics, and using them to study the metallicities of dwarf galaxies. In Section 6.2 below, we give some examples of the latter application, and show how the $\mathrm{H} \alpha$-detected galaxies may be useful in constraining the shape of the metallicity-luminosity relation.

Although discovered as part of the same survey, the highredshift, [O III]-detected galaxies are very different types of objects than the $\mathrm{H} \alpha$-selected galaxies. While the $\mathrm{H} \alpha$-selected objects are all dwarf galaxies, the [O III]-detected objects are a combination of luminous star-forming galaxies and AGNs. The star-forming galaxies at intermediate redshifts are particularly interesting because of their extremely low metallicities. These galaxies are similar in nature to the high-luminosity, lowmetallicity ELGs discovered by Salzer et al. (2009). The objects in this latter sample have very low abundances for their luminosities, as well as very high SFRs, suggesting that they are currently in an extreme stage of evolution. It has been suggested that they are undergoing their first major episode of star formation despite their short look-back times of 3-4 Gyr. The $\mathrm{H} \alpha$ dot selection technique has the potential to greatly enlarge the sample of these interesting objects. At the same time, the detection of strong-lined AGN at intermediate redshifts should improve our understanding of the populations of active galaxies at these redshifts, and allow us to directly measure the evolution of the AGN population between $\langle z\rangle \sim 0.33$ and today.

In this section, we present a preliminary analysis of some of the physical properties of this first list of objects detected in the $\mathrm{H} \alpha$ dot survey. In particular, we try to illustrate how some of the questions and problems mentioned above can be addressed using this sample of objects.

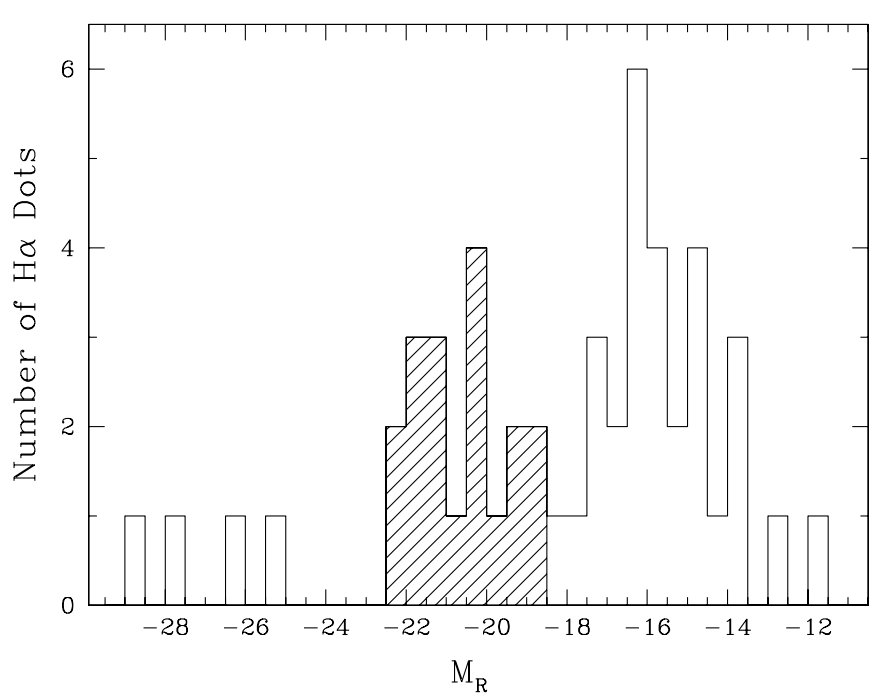

Figure 10. Histogram of $R$-band absolute magnitudes for the $\mathrm{H} \alpha$ dots. The lower-luminosity objects (no cross-hatching) are the $29 \mathrm{H} \alpha$-selected objects, while the cross-hatched portion of the histogram represents the [O III]-selected intermediate-redshift objects. There is no luminosity overlap between these two subsamples. The four QSOs are located on the far left with $M_{R}$ brighter than -25.0 .

\subsection{Absolute Magnitudes, $H \alpha$ Luminosities and Star-formation Rates}

There is perhaps no better way to visualize the diversity of the $\mathrm{H} \alpha$ dot sample than by looking at the distribution of the absolute magnitudes of its constituents. While it has become common to substitute stellar mass for luminosity in such comparisons, the lack of sufficient color data to do the calculations, plus the fact that the $\mathrm{H} \alpha$ dot sample is dominated by line emission and young stars, makes any conversion to stellar mass from luminosity dubious. Hence, we will restrict ourselves to looking at the $R$ band absolute magnitudes of the $51 \mathrm{H} \alpha$ dots that are bona fide emission-line objects. For this exercise we use the concordance cosmology of $H_{0}=70 \mathrm{~km} \mathrm{~s}^{-1} \mathrm{Mpc}^{-1}, \Omega_{m}=0.3$, and $\Omega_{\Lambda}=0.7$ to compute distances (which are tabulated in Table 4).

A histogram of absolute magnitudes is presented in Figure 10. The three primary types of $\mathrm{H} \alpha$ dots $(\mathrm{H} \alpha$-selected low-redshift dwarf galaxies, [O III]-selected intermediate-redshift objects, and high-redshift QSOs) are well separated in the diagram. We stress that no bandpass corrections ( $K$-corrections) have been applied to the [O III]-detected sources or the QSOs since we lack color information necessary to carry out such corrections. We note, however, that for the [O III]-detected objects the observed $R$-band flux corresponds closely to the rest-frame $B$-band. Hence, our values of $M_{R}$ for the [O III] subsample are more nearly consistent with rest-frame $M_{B}$. Since typical $B-R$ colors for star-forming galaxies are in the range of 1.0-1.5, their approximate $K$-corrected $R$-band absolute magnitudes would be shifted by this amount to the left (brighter absolute magnitudes) in Figure 10.

The nearby star-forming dwarfs are concentrated at the lowluminosity end of the diagram, covering the range -11.8 to -18.5 , with a median value of -15.9 . This median value is roughly two magnitudes fainter than the luminosity of the SMC, further underscoring their dwarf nature. The object with $M_{R}=-11.8(\mathrm{H} \alpha$ dot 11$)$ represents one of the lowestluminosity star-forming dwarf galaxies known. At the other extreme, the four QSOs all have absolute magnitudes brighter than -25 . The intermediate-redshift [O III]-detected objects 
Table 4

Derived Quantities

\begin{tabular}{|c|c|c|c|c|c|c|}
\hline $\begin{array}{l}\text { H } \alpha \text { Dot } \\
\text { (1) }\end{array}$ & $\begin{array}{c}\text { Distance } \\
(\mathrm{Mpc}) \\
(2)\end{array}$ & $\begin{array}{l}M_{R} \\
\text { (3) }\end{array}$ & $\begin{array}{c}\log \left(L_{\mathrm{H} \alpha}\right) \\
\left(\mathrm{erg} \mathrm{s}^{-1}\right) \\
(4)\end{array}$ & $\begin{array}{c}\mathrm{SFR} \\
\left(M_{\left.\odot \mathrm{yr}^{-1}\right)}\right. \\
(5)\end{array}$ & $\begin{array}{c}\Delta V \\
\left(\mathrm{~km} \mathrm{~s}^{-1}\right) \\
(6)\end{array}$ & $\begin{array}{c}\Delta R \\
(\mathrm{Mpc}) \\
(7)\end{array}$ \\
\hline 2 & 44.9 & -14.65 & 39.60 & 0.031 & 132 & 1.74 \\
\hline 3 & 45.3 & -14.06 & 39.44 & 0.022 & 99 & 1.31 \\
\hline 4 & 46.7 & -16.23 & 40.30 & 0.157 & $\ldots$ & $\ldots$ \\
\hline 5 & 1694.0 & -21.25 & $\ldots$ & $\ldots$ & $\ldots$ & $\ldots$ \\
\hline 6 & 1708.2 & -20.44 & $\ldots$ & $\ldots$ & $\ldots$ & $\ldots$ \\
\hline 7 & 1691.0 & -18.50 & $\ldots$ & $\ldots$ & $\ldots$ & $\ldots$ \\
\hline 8 & 48.8 & -17.54 & 39.25 & 0.014 & 208 & 3.01 \\
\hline 10 & 69.0 & -15.86 & 39.38 & 0.019 & 249 & 3.20 \\
\hline 11 & 38.8 & -11.77 & 38.54 & 0.003 & 55 & 0.50 \\
\hline 12 & 24.5 & -13.89 & 38.82 & 0.005 & 96 & 1.20 \\
\hline 13 & 27.5 & -13.96 & 38.67 & 0.004 & 69 & 1.00 \\
\hline 14 & 1713.1 & -22.33 & $\ldots$ & $\ldots$ & $\ldots$ & $\ldots$ \\
\hline 15 & 1784.3 & -21.41 & $\ldots$ & $\ldots$ & $\ldots$ & $\ldots$ \\
\hline 16 & 78.2 & -16.18 & 39.71 & 0.040 & 153 & 2.30 \\
\hline 17 & 9789.6 & -25.12 & $\ldots$ & $\ldots$ & $\ldots$ & $\ldots$ \\
\hline 18 & 54.5 & -15.32 & 39.27 & 0.015 & 221 & 3.50 \\
\hline 19 & 1693.4 & -20.18 & $\ldots$ & $\ldots$ & $\ldots$ & $\ldots$ \\
\hline 20 & 65.9 & -15.84 & 40.17 & 0.116 & 79 & 0.40 \\
\hline 21 & 1705.1 & -21.72 & $\ldots$ & $\ldots$ & $\ldots$ & $\ldots$ \\
\hline 22 & 1743.4 & -20.32 & $\ldots$ & $\ldots$ & $\ldots$ & $\ldots$ \\
\hline 24 & 1691.0 & -18.50 & $\ldots$ & $\ldots$ & $\ldots$ & $\ldots$ \\
\hline 27 & 29155.2 & -27.83 & $\ldots$ & $\ldots$ & $\ldots$ & $\ldots$ \\
\hline 28 & 1692.8 & -19.02 & $\ldots$ & $\ldots$ & $\ldots$ & $\ldots$ \\
\hline 30 & 1665.8 & -21.54 & $\ldots$ & $\ldots$ & $\ldots$ & $\ldots$ \\
\hline 31 & 65.0 & -15.00 & 39.71 & 0.040 & 39 & 0.22 \\
\hline 32 & 75.5 & -16.23 & 39.38 & 0.019 & 65 & 1.31 \\
\hline 33 & 60.2 & -16.32 & 39.34 & 0.017 & 1107 & 16.60 \\
\hline 34 & 75.5 & -16.94 & 40.01 & 0.081 & 42 & 0.11 \\
\hline 35 & 72.0 & -16.30 & 40.24 & 0.136 & 37 & 0.70 \\
\hline 36 & 29346.4 & -28.78 & $\ldots$ & $\ldots$ & $\ldots$ & $\ldots$ \\
\hline 37 & 1751.4 & -21.12 & $\ldots$ & $\ldots$ & $\ldots$ & $\ldots$ \\
\hline 38 & 1773.7 & -22.34 & $\ldots$ & $\ldots$ & $\ldots$ & $\ldots$ \\
\hline 39 & 74.2 & -14.89 & 39.46 & 0.023 & 124 & 2.40 \\
\hline 40 & 72.0 & -13.84 & 39.46 & 0.023 & 17 & 0.21 \\
\hline 41 & 72.5 & -18.47 & 40.80 & 0.496 & 8 & 0.71 \\
\hline 42 & 1757.6 & -21.96 & $\ldots$ & $\ldots$ & $\ldots$ & $\ldots$ \\
\hline 43 & 94.5 & -16.29 & 39.62 & 0.033 & $\ldots$ & $\ldots$ \\
\hline 44 & 108.3 & -16.88 & 40.33 & 0.167 & $\ldots$ & $\ldots$ \\
\hline 46 & 78.2 & -15.66 & 39.42 & 0.021 & $\ldots$ & $\ldots$ \\
\hline 47 & 76.4 & -12.58 & 39.46 & 0.023 & $\ldots$ & $\ldots$ \\
\hline 48 & 107.9 & -17.19 & 40.18 & 0.120 & $\ldots$ & $\ldots$ \\
\hline 49 & 77.3 & -16.00 & 39.81 & 0.052 & $\ldots$ & $\ldots$ \\
\hline 51 & 76.9 & -14.59 & 39.75 & 0.045 & $\ldots$ & $\ldots$ \\
\hline 52 & 21537.8 & -26.37 & $\ldots$ & $\ldots$ & $\ldots$ & $\ldots$ \\
\hline 53 & 103.4 & -17.50 & 40.69 & 0.391 & $\ldots$ & $\ldots$ \\
\hline 55 & 1806.1 & -18.90 & $\ldots$ & $\ldots$ & $\ldots$ & $\ldots$ \\
\hline 56 & 1809.8 & -20.87 & $\ldots$ & $\ldots$ & $\ldots$ & $\ldots$ \\
\hline 57 & 1810.5 & -19.53 & $\ldots$ & $\ldots$ & $\ldots$ & $\ldots$ \\
\hline 58 & 79.5 & -15.28 & 39.08 & 0.009 & $\ldots$ & $\ldots$ \\
\hline 59 & 1794.3 & -20.15 & $\ldots$ & $\ldots$ & $\ldots$ & $\ldots$ \\
\hline 60 & 87.0 & -17.04 & 39.62 & 0.033 & $\ldots$ & $\ldots$ \\
\hline 61 & 1829.8 & -19.27 & $\ldots$ & $\ldots$ & $\ldots$ & $\ldots$ \\
\hline
\end{tabular}

occupy the middle portion of the diagram, straddling the region around $L^{*}$. The full range of this subsample is -18.5 to -22.3 , with a median value of -20.7 . Recognizing that the rest-frame absolute magnitudes for the [O III]-detected subsample corresponds closely to $M_{B}$, this median absolute magnitude is approximately a half magnitude brighter than $M_{B} *$ (Faber et al. 2007). The six AGNs included in the [O III]selected subsample are preferentially at the brighter end of the distribution. In fact they represent six of the most luminous seven objects in the cross-hatched region of the histogram. The median $M_{R}$ for the [O III]-selected AGNs is -21.6, while the corresponding value for the 12 [O III]-selected star-forming galaxies is -20.2 -almost precisely the value for $M_{B} *$ in their rest frame.

The $\mathrm{H} \alpha$ luminosities of the $\mathrm{H} \alpha$-detected objects are calculated using the $\mathrm{H} \alpha$ fluxes measured from the narrowband images. 


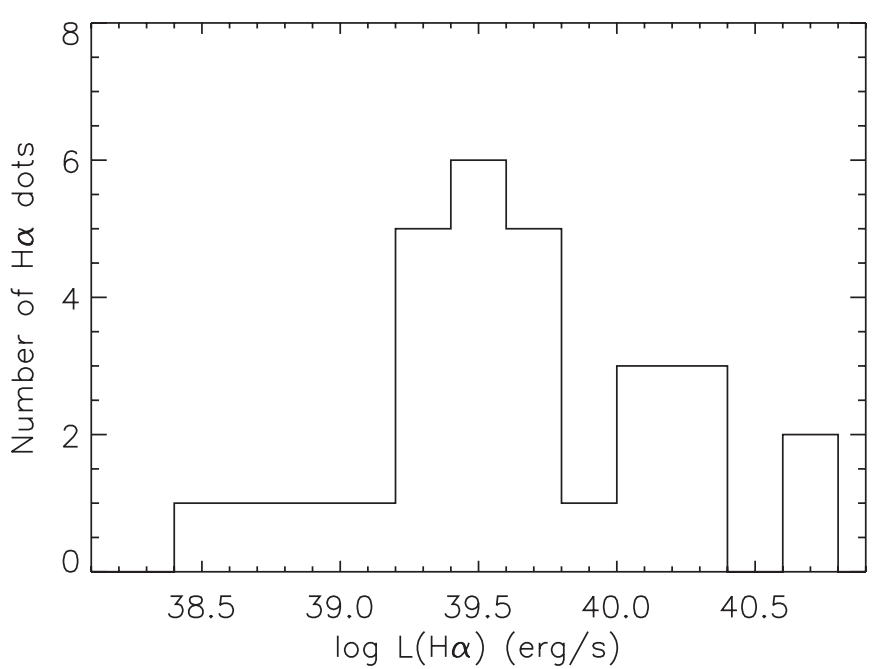

Figure 11. $\mathrm{H} \alpha$ luminosity distribution of the 29 low-redshift star-forming galaxies. The low median $\log \mathrm{H} \alpha$ luminosity (39.6) is consistent with the dwarf nature of the sample.

These are significantly more reliable than the spectroscopic fluxes, in large part due to the difficulty in centering some of the ultra-faint objects in the slit during the observations. For this calculation, we correct the observed $\mathrm{H} \alpha$ fluxes for the presence of $[\mathrm{N}$ II] emission in the filter bandpass using the spectroscopically measured $[\mathrm{N} \mathrm{II}] / \mathrm{H} \alpha$ ratios, as well as for Galactic and internal absorption using the spectroscopically derived values for $c_{\mathrm{H} \beta}$. Using the corrected $\mathrm{H} \alpha$ fluxes $\left(F_{\mathrm{H} \alpha}\right)$ and distances $(D)$, the $\mathrm{H} \alpha$ luminosity $\left(L_{\mathrm{H} \alpha}\right)$ is calculated using the following formula:

$$
L_{\mathrm{H} \alpha}=F_{\mathrm{H} \alpha} \cdot 4 \pi D^{2}
$$

A histogram of the $\mathrm{H} \alpha$ luminosities is shown in Figure 11. The $\mathrm{H} \alpha$ luminosities for this sample range from $3.4 \times 10^{38}$ to $6.3 \times 10^{40} \mathrm{erg} \mathrm{s}^{-1}$, with a median value of $3.9 \times 10^{39} \mathrm{erg} \mathrm{s}^{-1}$. The SFR for the $\mathrm{H} \alpha$-detected objects is calculated using the standard relation from Kennicutt (1998):

$$
\operatorname{SFR}\left(M_{\odot} \mathrm{yr}^{-1}\right)=7.9 \times 10^{-42} L_{\mathrm{H} \alpha}
$$

The SFRs of the $\mathrm{H} \alpha$-detected objects range from $0.003 M_{\odot} \mathrm{yr}^{-1}$ to $0.50 M_{\odot} \mathrm{yr}^{-1}$. For comparison, this falls within the range of SFRs $\left(10^{-5} M_{\odot} \mathrm{yr}^{-1}\right.$ to $\left.10 M_{\odot} \mathrm{yr}^{-1}\right)$ of the galaxies detected by the 11HUGS sample (Kennicutt et al. 2008). The median SFR is $0.031 M_{\odot} \mathrm{yr}^{-1}$, and a large majority (21 of 29 galaxies) have SFRs less than $0.05 M_{\odot} \mathrm{yr}^{-1}$, again attesting to the dwarf nature of the low-redshift subsample. Even though these objects are being selected due to their strong signatures of ongoing star formation, their typical SFRs are quite low. The total amount of star-formation activity represented in this entire sample only sums to $\sim 2.15 M_{\odot} \mathrm{yr}^{-1}$. The $\mathrm{H} \alpha$ luminosities and SFRs are tabulated for all $\mathrm{H} \alpha$-detected objects in Table 4.

\subsection{Abundances}

Metal abundances can be estimated for many of the starforming galaxies using standard "strong-line" methods (e.g., McGaugh 1991; Pilyugin 2000; Melbourne \& Salzer 2002; Kewley \& Dopita 2002; Salzer et al. 2005). Such methods utilize emission-line ratios such as $[\mathrm{O} \mathrm{III}] / \mathrm{H} \beta$, [N II $] / \mathrm{H} \alpha$, or $([\mathrm{O} \mathrm{III}]+[\mathrm{O} \mathrm{II}]) / \mathrm{H} \beta\left(=R_{23}\right)$ to provide coarse estimates of the metallicity of galaxies when a more precise analysis cannot

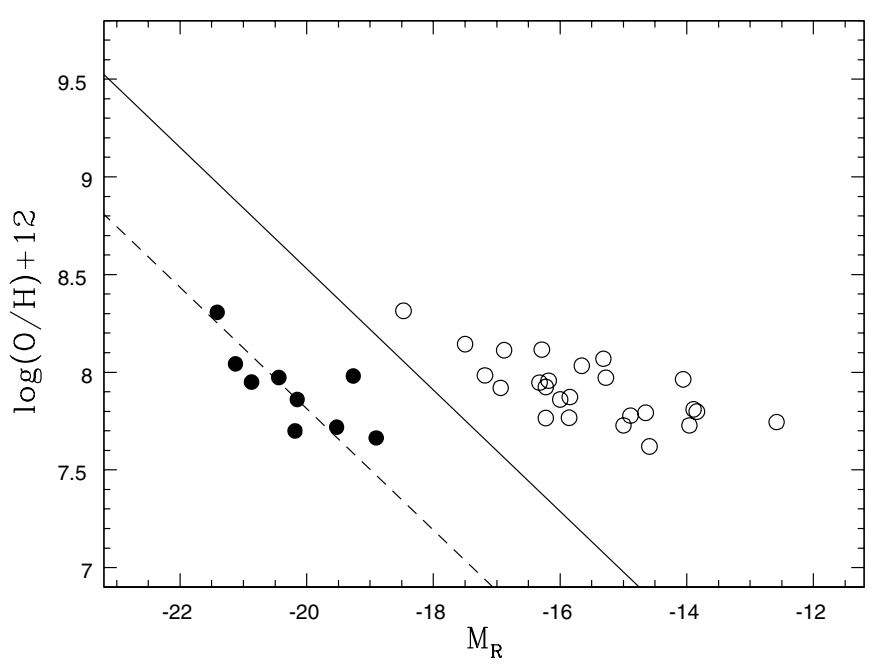

Figure 12. Luminosity-metallicity relationship for the star-forming galaxies from the $\mathrm{H} \alpha$ dot survey. The solid circles represent [O III]-detected objects, while the open circles are lower-redshift $\mathrm{H} \alpha$-detected galaxies. The solid line is the best-fit linear relation for the local sample of KISS galaxies found in Figure 3 of Salzer et al. (2009), offset to account for the difference in the filter used to derive the luminosity. The dashed line is the best-fit line through the [O III]-detected KISS galaxies from the same paper. The [O III]-detected $\mathrm{H} \alpha$ dots appear to follow the same $L-Z$ relation previously noted for the KISS galaxies.

be applied. For example, it is often the case that ELGs exhibit high signal-to-noise features from the stronger lines listed above while not having measurable temperature sensitive lines (e.g., [O III $]$ 24363) that are required for a full nebular abundance analysis. For the majority of ELGs, abundances can only be estimated using one of the strong-line methods.

We utilize the coarse abundance method developed by Salzer et al. (2005) for a large sample of ELGs from the KISS (Salzer et al. 2000, 2001) survey to estimate the oxygen abundances for the star-forming galaxies in the $\mathrm{H} \alpha$ dot survey. This method makes use of the $[\mathrm{O}$ III] $/ \mathrm{H} \beta$ and $[\mathrm{N}$ II $] / \mathrm{H} \alpha$ ratios. While it is less precise than many other abundance methods, it has the advantage of being simple and applicable to virtually all of the galaxies in the present sample since it is based on lines that are nearly always observed. The precision of the resulting abundances is $\sim 0.15-0.20$ dex.

Our abundance estimates are presented in Column 11 of Table 3 for those objects with the necessary spectral data. The majority of the star-forming galaxies have oxygen abundances $\log (\mathrm{O} / \mathrm{H})+12$ less than 8.00 (i.e., less than $\sim 20 \%$ solar, where we adopt 8.70 as the solar oxygen abundance (Scott et al. 2009)). The median value is 7.92 for the $34 \mathrm{H} \alpha$ dots listed. Nine of these are higher-redshift [O III]-detected objects; the range of observed abundances for the $\mathrm{H} \alpha$-detected and [O III]-detected sources is nearly identical, and both subsamples have the same mean metallicity of 7.91 .

The low average abundances of the $\mathrm{H} \alpha$-detected galaxies comes as no surprise, given their low-luminosity nature. It has been recognized for some time that low-luminosity/low-mass galaxies exhibit lower metallicities than their high-luminosity counterparts (e.g., Lequeux et al. 1979; Skillman et al. 1989; Tremonti et al. 2004; Salzer et al. 2005). Figure 12 shows a luminosity-metallicity $(L-Z)$ diagram for the current sample of star-forming galaxies. As in Figure 9, the $\mathrm{H} \alpha$-detected and [O III]-detected galaxies are plotted with different symbols. In this diagram, however, the two samples are quite distinct. The higher-redshift [O III]-detected galaxies are naturally found at higher luminosities, grouped roughly around $L^{*}$. 
The $\mathrm{H} \alpha$-detected galaxies represent low-luminosity starforming systems, as expected. While the two samples overlap completely in terms of their measured abundances, they are well separated in luminosity space.

The solid and dashed lines in Figure 12 both come from Figure 3 of Salzer et al. (2009). Since that figure used $B$-band luminosities, the solid line has been offset assuming a mean $B-R$ color for our dwarf star-forming galaxy sample of 1.3. However, the dashed line through the [O III]-selected galaxies has not been adjusted. As discussed above for the higher-redshift $\mathrm{H} \alpha$ dots the observed $R$-band is close to the rest-frame $B$-band, so the $B$-band relation from Salzer et al. (2009) fits the current sample quite well. While a proper comparison of the two data sets would normally require a full $K$-correction to the $R$-band luminosities, we do not currently possess adequate data to carry out the correction with suitable precision. Hence, for the current comparison we simply adopt the $B$-band fit to the KISS galaxies (which were appropriately $K$-corrected).

It would be possible for some of the low-luminosity dwarfs to appear at higher abundances if there were isolated extragalactic H II regions in our sample. These objects can be formed from gas pulled out of large, high-metallicity spiral galaxies (e.g., Ryan-Weber et al. 2004; Werk et al. 2008, 2010). Such sources could appear to be metal-rich for their luminosities. In the current sample of $\mathrm{H} \alpha$ dots only one object $(\mathrm{H} \alpha$ dot 60$)$ is classified as an outlying $\mathrm{H}$ II region (although it is not truly isolated from its parent galaxy), but in this case the host galaxy is a dwarf, so we do not see any outliers in the plot.

The offset between the two subsamples of $\mathrm{H} \alpha$ dots is nearly identical to the one found by Salzer et al. (2009) between a large sample of nearby $(z<0.09) \mathrm{H} \alpha$-detected KISS galaxies and a small number of [O III]-detected star-forming KISS objects in the redshift range $0.29-0.42$. While the two surveys differ in their selection methods (objective prism spectra versus narrowband filter excesses), the two samples of [O III]-selected objects appear to be analogous. The fact that the same $L-Z$ relation fits both the KISS and $\mathrm{H} \alpha$ dot samples is telling. We also note the similarities in the properties of our galaxies with the metal-poor star-forming galaxies highlighted in Hoyos et al. (2005) and the more luminous subset of the [O III]-detected objects described in Kakazu et al. (2007). The detection in the current survey of these metal-poor-yet-luminous star-forming systems at intermediate redshifts confirms the significance of this small but extremely interesting population of objects.

Finally, we note that the lower luminosity star-forming $\mathrm{H} \alpha$ dots appear to be significantly offset from the best-fit $L-Z$ relation of the KISS ELGs (Salzer et al. 2005, 2009). The reason for this most likely lies in the fact that the fit to the KISS $L-Z$ relationship is dominated by high-luminosity galaxies $\left(M_{R}\right.$ brighter than -19.0). As discussed in Salzer et al. (2005), samples dominated by higher luminosity galaxies tend to yield steeper $L-Z$ relations. It appears to be a common theme that dwarf galaxy samples yield best-fit $L-Z$ relations that are significantly shallower in slope (e.g., Skillman et al. 1989; Richer \& McCall 1995; Lee et al. 2003; Lee et al. 2004). Salzer et al. (2005) suggested the possibility that the $L-Z$ relation may not be strictly linear, but rather flattens out at low metallicities. While the current sample is too small to explore this issue, we note that it is entirely consistent with that hypothesis.

\subsection{Environment}

Clues about the nature of the $\mathrm{H} \alpha$ dots can be obtained by looking at the environments in which they are located. While we are not in a position to do this for the higher-redshift [O III]detected galaxies (this would require a redshift survey complete to $R \sim 21-22$ around each source), we can explore the local environments of the low-redshift $\mathrm{H} \alpha$-detected objects. As a first step in looking into environmental effects, we consider the separation distance between each $\mathrm{H} \alpha$-detected object and the ALFALFA galaxy that was the target of the narrowband observation. While comparing the locations of the $\mathrm{H} \alpha$-detected objects to the central ALFALFA galaxy in each image does not represent a comprehensive analysis of the environments of the $\mathrm{H} \alpha$-detected objects (such a study is beyond the scope of the current paper), it does allow us to get a sense for how clustered each $\mathrm{H} \alpha$-detected object is relative to what is usually the largest galaxy in each field.

We first recorded the positions and velocities of the ALFALFA target galaxy in each $\mathrm{H} \alpha$ dot field. Next, we computed the angular separation, velocity difference, and physical separation between each low-redshift $\mathrm{H} \alpha$-detected object and the ALFALFA galaxy in the field. For this work we excluded all $\mathrm{H} \alpha$ dots observed in the 2007 October run (numbers 43-61). This is because these fields were inadvertently observed with the incorrect narrowband filter (HA4 instead of HA3) during the observing run, due to an error made when the filters were loaded into the instrument. While the incorrect filter (HA4) was capable of detecting $\mathrm{H} \alpha$ dots, these objects were not in the same velocity range as the target ALFALFA galaxies (which should have been observed in HA3). Hence, carrying out the analysis described here would be both meaningless and misleading for these objects.

We start by comparing the distributions of the angular separations of $\mathrm{H} \alpha$ dots with the target ALFALFA galaxies for both the low-redshift and higher-redshift dots. As expected, the angular separations of the $\mathrm{H} \alpha$ dots that were not detected via their $\mathrm{H} \alpha$ line have a distribution consistent with random placement within the fields. On the other hand, the angular separations of the low-redshift $\mathrm{H} \alpha$-detected objects show a clear signature of being clustered toward the central ALFALFA galaxy. This suggests that there could be a physical connection between the low-redshift $\mathrm{H} \alpha$-detected objects and the nearby ALFALFA galaxies.

The possibility of a physical connection between the $\mathrm{H} \alpha$ detected objects and nearby ALFALFA galaxies is further supported by considering the velocity differences and physical separation distances. The width of each $\mathrm{H} \alpha$ filter is about $2000 \mathrm{~km} \mathrm{~s}^{-1}$. If there is no correlation between the $\mathrm{H} \alpha$-detected object and the ALFALFA galaxy, the velocity differences between them should be more or less randomly distributed. However, as can be seen in Figure 13, the majority of the $\mathrm{H} \alpha$ detected objects (17 of 18 , or $94 \%$ ) have velocity differences between them and the nearest ALFALFA galaxy of less than $250 \mathrm{~km} \mathrm{~s}^{-1}$. This suggests strongly that there tends to be a connection between the $\mathrm{H} \alpha$-detected object and the nearby ALFALFA galaxy. This impression is further strengthened by plotting the physical separations between the $\mathrm{H} \alpha$-detected objects and ALFALFA galaxies (Figure 14). The majority of $\mathrm{H} \alpha$-detected objects (12 of 18 , or $67 \%$ ) have physical separations of less than $2 \mathrm{Mpc}$, and for all but one the separation is under $4 \mathrm{Mpc}$. Many of the $\mathrm{H} \alpha$-detected galaxies appear to be sufficiently close to the ALFALFA target galaxy that they would be considered to be in the same group (Makarov \& Karachentsev 2011).

Three of the $\mathrm{H} \alpha$ dots $(39,40,41)$ appear not only to be connected with the nearby ALFALFA galaxy (UGC 10384), 


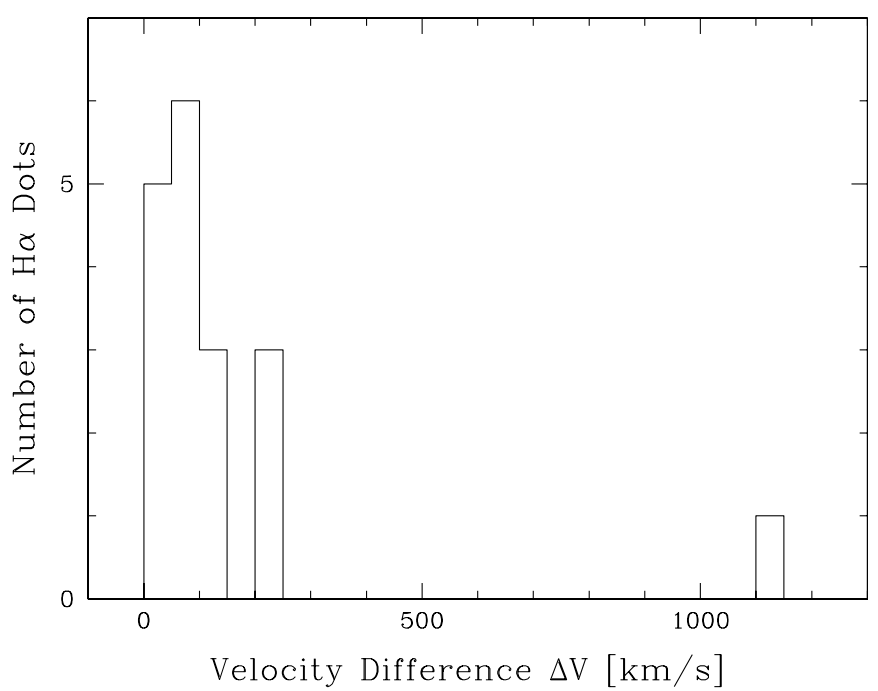

Figure 13. Absolute value of the velocity differences between each low-redshift $\mathrm{H} \alpha$ dot and the ALFALFA target galaxy from that field.

but they also appear to be connected with each other. They exhibit small angular separations, velocity differences, and physical separations between each other as well. All the velocity differences are less than $150 \mathrm{~km} \mathrm{~s}^{-1}$ and the physical separations are less than $2.2 \mathrm{Mpc}$. $\mathrm{H} \alpha$ dots 39 and 40 are illustrated in Figure 3 (top panel), where they are seen to be separated by only $\sim 10$ arcsec on the sky.

To summarize, the $\mathrm{H} \alpha$-detected objects show a strong tendency to cluster around the ALFALFA galaxy in each field. This is perhaps no surprise, since it has been known for some time that galaxies tend to be strongly clustered. On the other hand, the velocity range of the narrowband filters $\left(\sim 2000 \mathrm{~km} \mathrm{~s}^{-1}\right)$ is sufficiently large that one might have expected to see a larger spread in the observed velocity differences. The tendency toward very small velocity differences in Figure 13 illustrates the strength of this local clustering. We intend to explore this issue more rigorously and carefully in the future using a larger sample of $\mathrm{H} \alpha$ dots and a more comprehensive clustering analysis. Also, we plan to investigate whether there is a connection between the environment of the $\mathrm{H} \alpha$-detected galaxies and their star-formation properties. Ellison et al. (2010) find increased star formation in galaxy pairs in low-to-intermediate density environments. With a larger sample of $\mathrm{H} \alpha$-detected galaxies, we can explore how the star formation in the $\mathrm{H} \alpha$-detected galaxies is affected by the local and large-scale environment.

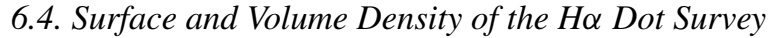

The measurement of the surface density of a survey is one (rather imperfect) indicator of the depth of the survey. The volume density of the detected objects can be compared with the volume density of other samples (e.g., the general galaxian luminosity function) to ascertain the contribution of the survey constituents to the overall population of galaxies in the same distance range. In this section, we present these numbers for this initial list of $\mathrm{H} \alpha$ dots, in an attempt to assess their significance relative to the general population.

The surface density of the $\mathrm{H} \alpha$ dot survey is calculated by simply dividing the number of $\mathrm{H} \alpha$ dots by the area of the sky covered by the survey. As detailed earlier, the effective area of our 205 narrowband images is $11.694 \mathrm{deg}^{2}$. Counting only the 51 unique dots that are bona fide emission-line sources, the

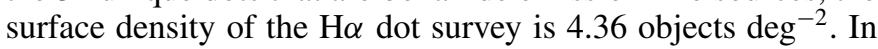

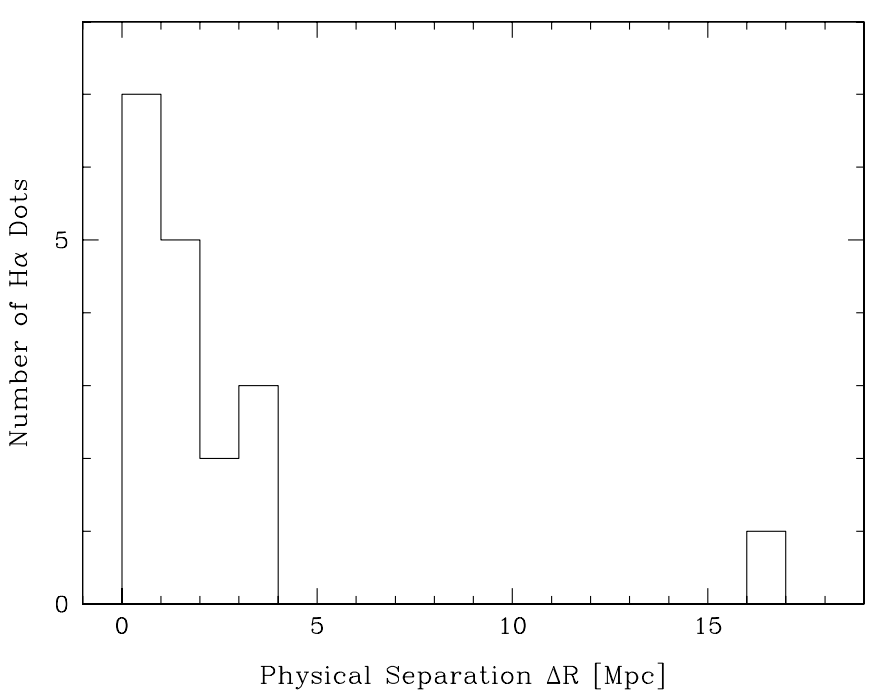

Figure 14. Derived physical separations between each low-redshift $\mathrm{H} \alpha$ dot and the ALFALFA target galaxy from that field.

order to gain insight into the depth and sensitivity of the $\mathrm{H} \alpha$ dot survey, it is interesting to compare this surface density to the density of other emission-line surveys. Perhaps the best survey to compare with is KISS, since like the $\mathrm{H} \alpha$ dot survey, it used a CCD as its detector (most previous ELG surveys were carried out with photographic plates). The overall surface density of the $\mathrm{H} \alpha$-selected KISS ELGs is $16.38 \mathrm{deg}^{-2}$ (Salzer et al. 2001; Gronwall et al. 2004; Jangren et al. 2005). However, since it is an objective-prism survey, the KISS survey covers a much larger redshift range than the $\mathrm{H} \alpha$ dot survey. For the KISS galaxies in the redshift range covered by the $\mathrm{H} \alpha$ dots $\left(1460-7810 \mathrm{~km} \mathrm{~s}^{-1}\right.$, which corresponds to the narrowband filters HA2-HA4) the surface density is $1.29 \mathrm{deg}^{-2}$ for the full velocity range, and only $\sim 1 / 3$ of this value if the velocity range of a single filter was used. So the KISS survey has the benefit of covering a much larger range in redshift $\left(0-28,500 \mathrm{~km} \mathrm{~s}^{-1}\right)$, while the $\mathrm{H} \alpha$ dot survey has the advantage of going deeper within a more restricted redshift range.

An even more telling statistic of the sensitivity of the $\mathrm{H} \alpha$ dot survey is the surface density of [O III]-detected objects. The 18 [O III]-selected dots have a surface density of $1.54 \mathrm{deg}^{-2}$. The KISS objective-prism method only detected 38 [O III]selected ELGs over the $147.6 \mathrm{deg}^{2}$ of the total survey, or 0.26 objects $\mathrm{deg}^{-2}$. Correcting for the differences in the wavelength coverages of the two surveys ( $800 \AA$ for KISS and $70 \AA$ for the $\mathrm{H} \alpha$ dot survey) reduces the effective surface density of the

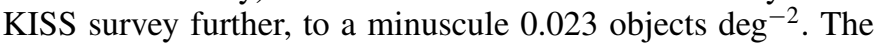
narrowband survey method is much more effective at finding the faint but strong-lined objects at intermediate redshifts than was KISS.

We can also calculate approximate volume densities of the $\mathrm{H} \alpha$ dots. The volume density of the low-redshift $\mathrm{H} \alpha$ dots is calculated by dividing the number of objects by the effective volume of the survey in the redshift range covered by the narrowband filters $\left(\sim 1720 \mathrm{Mpc}^{3}\right)$. However, the effective volume is actually smaller still, since each field is imaged through only one filter, and hence the redshift range covered is only about a third of the total value covered by HA2-HA4. If we do the calculation only for objects detected in filter HA3 $(N=16)$, the effective volume is $\sim 462 \mathrm{Mpc}^{3}$, which yields an approximate volume density of 0.035 low-redshift $\mathrm{H} \alpha$ dots per $\mathrm{Mpc}^{3}$. This can be compared to the volume density for all dwarf 
galaxies of $\sim 0.22$ galaxies $\mathrm{Mpc}^{-3}$, derived by integrating the local $R$-band luminosity function of Geller et al. (1997) between $M_{R}-17.5$ and -13.5 , the approximate luminosity range of the low-redshift $\mathrm{H} \alpha$ dots. Hence, the $\mathrm{H} \alpha$ dots appear to represent $\sim 16 \%$ of the dwarf galaxy population outside of clusters. This number is highly uncertain, but suggests that the low-redshift $\mathrm{H} \alpha$ dots represent a significant component of the overall dwarf galaxy population.

The volume density of [O III]-selected star-forming galaxies, computed in the same fashion, is $4.7 \times 10^{-5} \mathrm{Mpc}^{-3}$, while the density of [O III]-selected AGN is $2.3 \times 10^{-5} \mathrm{Mpc}^{-3}$. These latter values can be compared directly with numbers for the [O III]-selected KISS galaxies (Salzer et al. 2009), who found a volume density of $4.3 \times 10^{-7} \mathrm{Mpc}^{-3}$ for the star-forming galaxies and $6.6 \times 10^{-7} \mathrm{Mpc}^{-3}$ for the AGNs. Hence, we see that the $\mathrm{H} \alpha$ dot selection method is much more sensitive to emissionline sources at these redshifts than was the KISS objective-prism method. This was hinted at above, but the volume densities presented here drive home the point quite dramatically. It is worth stressing, however, that the [O III]-selected $\mathrm{H} \alpha$ dots still represent only a small fraction of all galaxies in their luminosity range at these redshifts. The overall volume density of galaxies with comparable luminosities in the general luminosity function of Faber et al. (2007) in the same redshift range is $\sim 2.5 \times$ $10^{-3} \mathrm{Mpc}^{-3}$.

\section{SUMMARY AND CONCLUSIONS}

We present the first installment of the $\mathrm{H} \alpha$ dot survey. This project is enabled by using existing narrowband images, taken for a different survey program, and searching the processed data for point sources of line emission. In that sense, it is purely a "spin-off" project, utilizing pre-existing data to carry out serendipitous science.

Using our own software package, we have searched 205 fields imaged through narrowband filters for point sources of line emission. We have cataloged 61 objects (60 unique sources) in a survey area of $11.694 \mathrm{deg}^{2}$. The search method selects faint objects (median $R$ magnitude $=19.5$, with the faintest object at $R=22.6$ ) with moderate-to-faint line fluxes (as low as $6.1 \times$ $10^{-16} \mathrm{erg} \mathrm{s}^{-1} \mathrm{~cm}^{-2}$ ). Follow-up spectra of the full sample reveal that the dots are a mixture of low-redshift $\mathrm{H} \alpha$-detected dwarf galaxies (29), intermediate-redshift [O III]-detected galaxies (18), high-redshift QSOs (4), and false detections (9). We present a preliminary analysis of the properties of the $\mathrm{H} \alpha$ dots, including their broadband and $\mathrm{H} \alpha$ luminosities, SFRs, metal abundances, and clustering properties.

Throughout the paper, we have attempted to illustrate specific areas of research connected to the study of galaxy evolution where the $\mathrm{H} \alpha$ dots could be utilized to good advantage. It should be immediately clear from the selection method that the $\mathrm{H} \alpha$ dots do not represent a comprehensive sample of starforming galaxies or AGNs. However, the selection method does provide unique samples of objects in specific redshift windows that allow for the detailed study of interesting sub-populations of objects.

Low-luminosity star-forming dwarf galaxies. As we have stressed throughout this paper, the $\mathrm{H} \alpha$-detected objects cataloged in our survey represent very-low-luminosity galaxies with active star formation. While this does not necessarily mean that the objects presented here are either the most actively starforming dwarfs nor the very-lowest-luminosity star-forming galaxies, we believe that they constitute a representative sample of low-luminosity actively star-forming galaxies. They span a luminosity range that does extend down to the lowest luminosities populated by known star-forming dwarfs $\left(M_{R} \sim-12\right)$. The current sample includes several excellent candidates for more detailed metallicity studies using high-quality spectra. The narrowband selection method appears to be effective at picking out interesting dwarf galaxies over the full range of redshifts covered by our narrowband filters.

Low-metallicity $L^{*}$ galaxies at intermediate redshifts. The first installment of the $\mathrm{H} \alpha$ dot survey has discovered a dozen star-forming galaxies via their [O III] emission at intermediate redshifts $(z=0.32-0.34)$. These galaxies are extremely metal poor despite having luminosities comparable to $L^{*}$. Like the luminous but metal-poor objects discovered previously by Kakazu et al. (2007) and Salzer et al. (2009), these systems appear to be in an unusual evolutionary state. As stressed in Salzer et al., these galaxies may be undergoing their first major episode of star formation at a look-back time of 3-4 Gyr. The $\mathrm{H} \alpha$ dot survey method provides an efficient technique for discovering additional examples of these enigmatic galaxies, which may hold important clues to galaxy formation processes that are accessible for study at modest redshifts.

Seyfert galaxies at intermediate redshifts. The $\mathrm{H} \alpha$ dot survey has also discovered a number of [O III]-selected AGNs, mostly Seyfert 2 galaxies, but also one Seyfert 1.9 and a borderline LINER/Seyfert 2. The narrowband excess selection method appears to make the detection of intermediate-redshift AGNs accessible to fairly low line fluxes and equivalent widths, as evidenced by the detection of $\mathrm{H} \alpha$ dot 30 . This suggests that this method can be used to develop a fairly robust sample of AGNs in the $z=0.32-0.34$ range. While the current number of $\mathrm{H} \alpha$ dot AGN is small (six), we can expect that number to increase significantly as the survey continues.

Isolated extragalactic $H$ II regions. Following on the studies of Ryan-Weber et al. (2004) and Werk et al. (2010), we expected to detect at least a few examples of this class of emission-line object. In hindsight, however, we perhaps should not be too surprised by our lack of detections. Given the differences in the SINGG and ALFALFA samples, plus the fact that we purposely avoided observing low-redshift objects in the ALFALFA $\mathrm{H} \alpha$ project (velocities less than $\sim 1500 \mathrm{~km} \mathrm{~s}^{-1}$ ), the current project has relatively few large, extended spirals of the type where the extragalactic H iI regions found by Ryan-Weber et al. and Werk et al. were located. Furthermore, while detecting such objects was the primary goal of these previous studies, our focus was on detecting a more eclectic group of emission-line objects. Our ongoing survey project has in fact detected several excellent candidate isolated extragalactic $\mathrm{H}$ II regions, which will be reported in a subsequent survey paper.

We have already started to explore other aspects of the $\mathrm{H} \alpha$ dots, including obtaining multi-wavelength data for a number of objects. For example, we have obtained deep near- and farUV GALEX images of several of the $\mathrm{H} \alpha$-detected objects. We will use these data to measure the UV SFRs of the H $\alpha$-detected objects, search for XUV disks, and try to use these data to help distinguish between isolated extragalactic $\mathrm{H}$ II regions and dwarf galaxies. In addition, we have obtained $\mathrm{C}$ and D-array H I $21 \mathrm{~cm}$ maps of some of the $\mathrm{H} \alpha$-detected objects using the VLA (Allan et al. 2010). The $21 \mathrm{~cm}$ maps will allow us to determine whether the $\mathrm{H} \alpha$-detected objects are emissionline sources embedded in an extended $\mathrm{H}$ I envelop surrounding the ALFALFA galaxy, whether they are located within tidally disturbed gas, or whether they are completely independent dwarf galaxies located in proximity to the ALFALFA source. 
The continuation of the $\mathrm{H} \alpha$ dot survey will lead to the detection of many more interesting objects. We have currently cataloged more than 200 sources, and are continuing our efforts to obtain follow-up spectra for all of the $\mathrm{H} \alpha$ dot candidates. A preliminary report on this extended sample of dots was given by Feddersen et al. (2011). We hope to complete our spectral observations for this new group of dots shortly, and be able to present our extended sample along with a complete analysis of the new sources in the near future.

J.J.S. gratefully acknowledges funding from the National Science Foundation through award AST-0823801 that supports the ALFALFA $\mathrm{H} \alpha$ project. In addition, we express our thanks to Wesleyan University and the College of Arts and Sciences at Indiana University for their support of the WIYN $0.9 \mathrm{~m}$ telescope that was used to obtain the narrowband images necessary for the survey, and also to Dartmouth College for their support of the MDM Observatory. This project was conceived as an undergraduate research program, and all of the student participants at both Wesleyan University and Indiana University have been (and continue to be) undergraduates. Some of the ALFALFA $\mathrm{H} \alpha$ observations used in this study were obtained by Wesleyan University undergraduates Arthur Sugden, Matthew Johnson, Seth Cohen, and Christopher Dieck, with assistance from Ed Moran and John Cannon. The software used for the basic processing of the $\mathrm{H} \alpha$ images was written largely by Arthur Sudgen. J.A.K. thanks Christopher Dieck for useful conversations about unusual emission in one of the $\mathrm{H} \alpha$ images. The Marcario Low Resolution Spectrograph at the HET is named for Mike Marcario of High Lonesome Optics, who fabricated several optics for the instrument but died before its completion. The LRS is a joint project of the Hobby-Eberly Telescope partnership and the Instituto de Astronoma de la Universidad Nacional Autnoma de México.

\section{REFERENCES}

Allan, J., Cannon, J. M., Kellar, J., Salzer, J. J., \& Rosenberg, J. L. 2010, BAAS, 42,482

Baldwin, J. A., Phillips, M. M., \& Terlevich, R. 1981, PASP, 93, 5

Boquien, M., Duc, P.-A., Braine, J., et al. 2007, A\&A, 467, 93

Boroson, T. A., Salzer, J. J., \& Trotter, A. 1993, ApJ, 412, 524

Dopita, M. A., \& Evans, I. N. 1986, ApJ, 307, 431

Dunkley, J., Komatsu, E., Nolta, M. R., et al. 2009, ApJS, 180, 306

Ellison, S., Patton, D., Simard, L., et al. 2010, MNRAS, 407, 1514

Faber, S. M., Willmer, C. N. A., Wolf, C., et al. 2007, ApJ, 665, 265
Feddersen, J., Salzer, J. J., Williams, A., \& Gronwall, C. 2011, BAAS, 43, 54 Ferguson, A., Wyse, R., Gallagher, J., \& Hunter, D. 1998, ApJ, 506, 19 Geller, M. J., Kurtz, M. J., Wegner, G., et al. 1997, AJ, 114, 2205

Giovanelli, R., Haynes, M. P., Kent, B. R., et al. 2005, AJ, 130, 2598

Gronwall, C., Salzer, J. J., Sarajedini, V. L., et al. 2004, AJ, 127, 1943

Haynes, M. P., Giovanelli, R., Martin, A. M., et al. 2011, AJ, 142, 170

Hoyos, C., Koo, D. C., Phillips, A. C., Willmer, C. N. A., \& Guhathakurta, P. 2005, ApJ, 635, L21

Hill, G. J., Nicklas, H. E., MacQueen, P. J., et al. 1998, Proc SPIE, 3355, 375

Jangren, A., Salzer, J. J., Sarajedini, V. L., et al. 2005, AJ, 130, 2571

Kakazu, Y., Cowie, L. L., \& Hu, E. M. 2007, ApJ, 668, 853

Kauffmann, G., Heckman, T. M., Tremonti, C., et al. 2003, MNRAS, 346, 1055

Kennicutt, R. C. 1998, ARA\&A, 36, 189

Kennicutt, R. C., Lee, J. C., Funes, J. G., Sakai, S., \& Akiyama, S. 2008, ApJS, 178,247

Kewley, L. J., \& Dopita, M. A. 2002, ApJS, 142, 35

Kewley, L. J., Dopita, M. A., Sutherland, R. S., Heisler, C. A., \& Trevena, J. 2001, ApJ, 556, 121

Lee, H., McCall, M. L., Kingsburgh, R. L., Ross, R., \& Stevenson, C. C. 2003, AJ, 125, 145

Lee, J. C., Kennicutt, R. C., Funes, J. G., Sakai, S., \& Akiyama, S. 2007, ApJ, $671, \mathrm{~L} 113$

Lee, J. C., Kennicutt, R. C., Funes, J. G., Sakai, S., \& Akiyama, S. 2009, ApJ, 692, 1305

Lee, J. C., Salzer, J. J., \& Melbourne, J. 2004, ApJ, 616, 752

Lequeux, J., Rayo, J. F., Serrano, A., Peimbert, M., \& Torres-Peimbert, S. 1979, A\&A, 80, 155

Makarov, D., \& Karachentsev, I. 2011, MNRAS, 412, 2498

Martin, A., Giovanelli, R., Haynes, M. P., \& Guzzo, L. 2012, ApJ, 750, 38

Martin, A., Papastergis, E., Giovanelli, R., et al. 2010, ApJ, 723, 1359

Massey, P., Strobel, K., Barnes, J. V., \& Anderson, E. 1988, ApJ, 328, 315

Meurer, G. R., Hanish, D. J., Ferguson, H. C., et al. 2006, ApJS, 165, 307

McGaugh, S. S. 1991, ApJ, 380, 140

Melbourne, J., \& Salzer, J. J. 2002, AJ, 123, 2302

Oke, J. B., \& Gunn, J. E. 1983, ApJ, 266, 713

Osterbrock 1989, Astrophysics of Gaseous Neblae and Active Galactic Nuclei (Mill Valley, CA: University Science Books)

Pilyugin, L. S. 2000, A\&A, 362, 325

Richer, M. G., \& McCall, M. L. 1995, ApJ, 445, 642

Ryan-Weber, E. V., Meurer, G. R., Freeman, K. C., et al. 2004, AJ, 127, 1431

Salzer, J. J., Gronwall, C., Lipovetsky, V. A., et al. 2000, AJ, 120, 80

Salzer, J. J., Gronwall, C., Lipovetsky, V. A., et al. 2001, AJ, 121, 66

Salzer, J. J., Lee, J. C., Melbourne, J., et al. 2005, ApJ, 624, 661

Salzer, J. J., Williams, A. L., \& Gronwall, C. 2009, ApJ, 695, L67

Scott, P., Asplund, M., Grevesse, N., \& Sauval, A. J. 2009, ApJ, 691, 119

Skillman, E. D., Kennicutt, R. C., \& Hodge, P. W. 1989, ApJ, 347, 875

Sugden, A., Salzer, J., Kellar, J., et al. 2008, BAAS, 40, 215

Thilker, D. A., Bianchi, L., Meurer, G., et al. 2007, ApJS, 173, 538

Tremonti, C. A., Heckman, T. M., Kauffmann, G., et al. 2004, ApJ, 613, 898

van Dokkum, P. 2001, PASP, 113, 1420

Veilleux, S., \& Osterbrock, D. E. 1987, ApJS, 63, 295

Werk, J. K., Putman, M. E., Meurer, G. R., et al. 2008, ApJ, 678, 888

Werk, J. K., Putman, M. E., Meurer, G. R., et al. 2010, AJ, 139, 279

White, R., Becker, R. H., Gregg, M. D., et al. 2000, ApJS, 126, 133 